\title{
Sheath vaporization of a monodisperse fuel-spray jet
}

\author{
J. ARRIETA-SA NA GUSTÍN,A.L.SÁNCHEZ, A. LIÑ ÁN \\ AND F. A. WILLIA MS
}

The group vaporization of a monodisperse fuel-spray jet discharging into a hot coflowing gaseous stream is investigated for steady flow by numerical and asymptotic methods with a two-continua formulation used for the description of the gas and liquid phases. The jet is assumed to be slender and laminar, as occurs when the Reynolds number is moderately large, so that the boundary-layer form of the conservation equations can be employed in the analysis. Two dimensionless parameters are found to control the flow structure, namely the spray dilution parameter $\lambda$, defined as the mass of liquid fuel per unit mass of gas in the spray stream, and the group vaporization parameter $\varepsilon$, defined as the ratio of the characteristic time of spray evolution due to droplet vaporization to the characteristic diffusion time across the jet. It is observed that, for the small values of $\varepsilon$ often encountered in applications, vaporization occurs only in a thin layer separating the spray from the outer droplet-free stream. This regime of sheath vaporization, which is controlled by heat conduction, is amenable to a simplified asymptotic description, independent of $\varepsilon$, in which the location of the vaporization layer is determined numerically as a free boundary in a parabolic problem involving matching of the separate solutions in the external streams, with appropriate jump conditions obtained from analysis of the quasi-steady vaporization front. Separate consideration of dilute and dense sprays, corresponding, respectively, to the asymptotic limits $\lambda \ll 1$ and $\lambda \gg 1$, enables simplified descriptions to be obtained for the different flow variables, including explicit analytic expressions for the spray penetration distance.

\section{Introduction}

Because of its relevance in many industrial applications, the combustion and vaporization of fuel sprays has been the subject of many previous investigations (see e.g. Faeth 1983; Sirignano 1983, 1999; Williams 1985; Annamalai \& Ryan 1992; Crowe, Sommerfeld \& Tsuji 1998 for reviews of the early work). Although the initial studies focused on the response of isolated droplets, leading to expressions for the 
vaporization, relative motion and burning rate of individual droplets (Williams 1985), it was early recognized that in many practical situations, fuel sprays evaporate or burn as a group (Chiu \& Liu 1977; Labowsky \& Rosner 1978; Correa \& Sichel $1982 a, b)$. Different regimes of fuel-spray group combustion were identified, including cases where the diffusion flame lies outside the droplet cloud; there, the fuel that originates from the vaporizing droplets burns with the ambient oxygen, with droplet vaporization occurring either all throughout the cloud (external group combustion) or in a thin outer layer on the outer edge of the droplet cloud (external sheath combustion). It was also seen that individual droplet combustion may also occur, provided the spray is sufficiently dilute, with oxygen diffusing across the resulting cloud of burning droplets, each one of them being surrounded by a closed flame if their radius is large enough to sustain the flame. Besides these combustion modes, for a narrow range of conditions, there exists a transition regime termed internal group combustion (Chiu, Kim \& Croke 1982), in which an internal diffusion flame separates a group of vaporizing droplets from a group of individually burning droplets, a configuration that has been observed in laboratory experiments (see e.g. Chen \& Gomez 1997; Russo \& Gomez 2006). As mentioned in Chiu et al. (1982), experimental evidence suggests that group combustion is the predominant form of spray combustion in typical industrial burners.

Progress in understanding of spray vaporization and combustion relies on advanced diagnostic techniques (Chen \& Gomez 1997; Karpetis \& Gomez 2000; Russo \& Gomez 2006) as well as on increased computer power, which enables, for instance, analyses of droplet array combustion to be advanced well beyond the initial analytic efforts (Labowsky 1980), an example being the recent computation of heptane-droplet group combustion in a staggered configuration of Lee et al. (2010). With the present computer power, direct numerical simulations of combustion of turbulent sprays at moderate Reynolds numbers are feasible (Reveillon \& Vervisch 2005; Luo et al. 2011), and more complex computations including detailed chemistry and higher Reynolds numbers can be envisioned in the near future. While numerical approaches can include many phenomena, asymptotic and analytic methods, such as those employed here, are better suited for isolating the most important effects, thereby increasing understanding of the underlying physics significantly. In addition, they often can yield formulae that are readily applied to calculate quantities of interest in applications.

Although in many combustors the fuel is introduced in the combustion chamber as a high-velocity swirling liquid jet that breaks up to form a fuel-spray jet (Luo et al. 2011), the jet configuration has been subject to a limited number of theoretical investigations (Chiu et al. 1978; Kim \& Chiu 1983). Instead, most of the initial analyses considered vaporization (Correa \& Sichel 1982a) or combustion (Chiu \& Liu 1977; Labowsky \& Rosner 1978; Correa \& Sichel 1982 b) of a spherical droplet cloud, with the objective of gaining insight into the underlying competing physical phenomena rather than evaluating a specific practical application.

The process of liquid jet atomization is highly complex, with the effects of injector boundary layers, droplet breakup and collision, turbulence and recirculation playing key roles in determining the characteristics of the resulting spray (Lasheras \& Hopfinger 2000). Since the liquid density is typically a factor up to $10^{3}$ larger than the gas density, appreciable liquid heating and vaporization resulting from heat transfer from the gas carrier occurs only far from the injection region, once the spray stream becomes sufficiently dilute for the liquid phase to occupy a small volumetric fraction, of the order of $10^{-3}$. The processes of liquid-jet atomization leading to spray formation and that of spray vaporization therefore occur in separate spatial regions, 


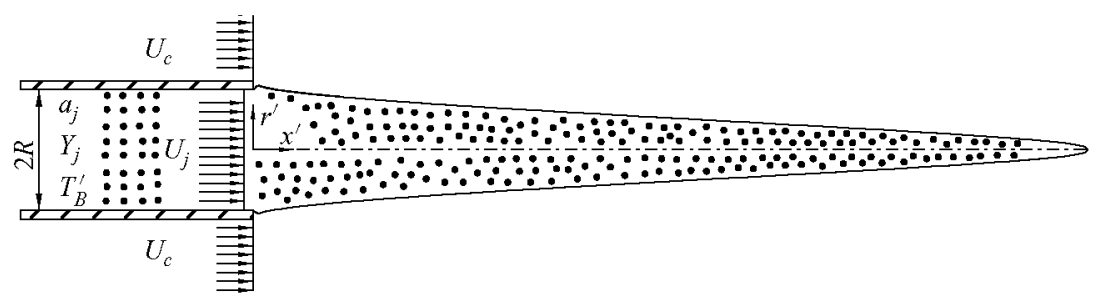

FIGURE 1. Vaporization of a monodisperse spray.

and can be consequently studied independently, with the latter being the subject of the present investigation.

To focus more directly on the group vaporization process, a simple laminar configuration including a central monodisperse fuel-spray jet discharging with a high Reynolds number into a surrounding hot coflow, sketched in figure 1, is selected for the study, the objective being that of developing new understanding, which is also sought in recent experimental studies involving laminar sprays (Chen \& Gomez 1997; Karpetis \& Gomez 2000; Russo \& Gomez 2006). Clearly, given the simplicity of the flow considered, the results cannot be expected to be directly applicable to realistic configurations such as transient diesel sprays, supercritical conditions or complex turbulent flows in gas turbines with potential acoustic amplification of pressure oscillations, but can help in developing ideas for these applications. For instance, key controlling parameters will be identified and their influence on the spray structure will be described both numerically and analytically. Particular attention will be given to the sheath-vaporization regime, previously analysed by Correa \& Sichel (1982a) for the spherical droplet cloud, with droplet vaporization occurring only in a thin layer surrounding the spray, whose location will be found as a free boundary in a parabolic problem that is solved by numerical integration in the distinguished regime $\lambda \sim O(1)$, with $\lambda$ representing the mass of liquid fuel per unit mass of gas in the spray stream, as defined below in (3.4).

The description of the spray dynamics is facilitated by the disparity of length scales often encountered in realistic applications. Thus, in sprays with a large number of droplets, the characteristic transverse spray size, given in our case by the injector radius $R$, is much larger than the average distance between neighbouring droplets $l_{d}=n_{j}^{-1 / 3}$, with $n_{j}$ denoting the number of droplets per unit volume at the jet exit. The other relevant distance involved in the analysis is the characteristic droplet size, given by the value of the droplet radius at the jet exit $a_{j}$, which is much smaller than $l_{d}$ in dilute sprays. The inequalities

$$
R \gg l_{d} \gg a_{j}
$$

are therefore expected to hold for dilute sprays with many droplets, found in most spray applications.

Because of the condition $a_{j} \ll l_{d}$, each droplet vaporizes and moves with no direct effects from neighbouring droplets. Thus, the main effects on the vaporization of the droplets are not due to the direct influence of their neighbours, but are associated with the mean gas-phase collective environment created by all the droplets. Each droplet produces relatively large variations to the composition and temperature field that are felt only in the immediate vicinity of the droplet, decaying at distances of the order of $a_{j}$, so that in the gas phase between droplets the variations in the different properties are much smaller. The description of these slow variations of the different gas-phase 
variables, including the velocity, temperature, density and relevant mass fractions, which occur over distances of the order of $R$, can be carried out at any spatial point by space-averaging over a neighbourhood of that point of size $L$, with $L$ in the range $R \gg L \gg l_{d}$. The vaporizing droplets appear as point sources of mass and momentum and point sinks of heat when seen with the scale $L$, with the vaporization rate of and the force acting on each individual droplet determined as those of the isolated droplet surrounded by the mean local environment. Since $L \gg l_{d}$, each computational cell includes many droplets, and the corresponding point sources appear as distributed.

While a Eulerian description emerges naturally for the gas phase, the liquid phase is in principle more easily described with a Lagrangian approach in which each droplet is traced individually, with the ambient properties changing as the droplet moves across the flow field. This Eulerian-Lagrangian approach is widely used in computations of turbulent flows, an example being the particle-source-in-a-cell model of typical turbulent combustion codes. An application of this combined EulerianLagrangian modelling strategy can be found for instance in the formulation recently proposed by Bermúdez, Ferrín \& Liñán (2007) for the description of group combustion in pulverized coal furnaces.

An alternative formulation is possible, in which the liquid phase is also treated as a continuum. In the resulting two-continua formulation (Sirignano 1999), the droplet population is described in terms of the number of droplets per unit volume through a conservation equation. Although this Eulerian-Eulerian approach is often simpler and greatly facilitates analytical work, it is only well suited for the treatment of monodisperse laminar sprays, as the one considered here, whereas in the presence of crossing droplet trajectories, as occurs in turbulent flow or with recirculating flow regions when the particle size is not small enough, this continuum description fails, and tracking of individual droplets becomes necessary.

This paper is organized as follows. In the next section, the problem definition will be given, followed in $\S 3$ by a discussion of the relevant time scales and resulting controlling parameters. The dimensionless formulation of the group-vaporization problem is presented in $\$ 4$, and sample numerical integrations are offered in $\S 5$. The subsequent sections are specifically devoted to the limit of sheath vaporization. The formulation of the associated free-boundary problem is provided in $\S 6$, with sample numerical results shown in $\$ 7$. The treatments of the asymptotic limits of dense and dilute sprays are presented in $\$ \S 8$ and 9 , respectively. The final section is devoted to concluding remarks.

\section{Problem statement}

The jet spray includes the interaction region between the round spray flowing out of the injector and the hot coflowing stream at temperature $T_{c}^{\prime}$ larger than that of the injected jet, $T_{j}^{\prime}$. The velocity profiles in the jet and the coflow are assumed to be uniform, with values given respectively by $U_{j}$ and $U_{c}$. The spray is assumed to be monodisperse, with the uniform values $a_{j}$ and $n_{j}$ of the droplet radius and the droplet number density at the jet exit. Furthermore, the injector is assumed to be sufficiently long for the mixture to be in saturated equilibrium at the jet exit. Thus, the temperature $T_{j}^{\prime}$ and initial fuel mass fraction $Y_{j}$ in the jet stream can be related by the Clausius-Clapeyron equation

$$
Y_{j}=\frac{W_{F}}{W_{j}} \exp \left[L_{v} /\left(R_{F} T_{B}^{\prime}\right)-L_{v} /\left(R_{F} T_{j}^{\prime}\right)\right],
$$


where $T_{B}^{\prime}$ is the boiling temperature, $L_{v}$ is the specific latent heat of vaporization, $R_{F}=R^{o} / W_{F}$ is the gas constant of the fuel, with $R^{o}$ representing the universal gas constant, and $W_{F}$ and $W_{j}$ are the molecular weight of the fuel and the jet carrier gas mixture, respectively. It is assumed that, as often occurs in applications, $L_{v} /\left(R_{F} T_{B}^{\prime}\right) \gg 1$, which implies that, at equilibrium, the departures of $T_{j}^{\prime}$ from the boiling temperature $T_{B}^{\prime}$ are of order $T_{j}^{\prime}-T_{B}^{\prime} \sim\left[L_{v} /\left(R_{F} T_{B}^{\prime}\right)\right]^{-1} T_{B}^{\prime} \ll T_{B}^{\prime}$, and can be consequently neglected in the first approximation.

We assume in our analysis that the spray is dilute (in the sense that the volume fraction of the liquid is small, even though we consider the liquid mass per unit volume to be comparable with or larger than that of the gas) and that the spray contains many droplets, so that the inequalities given in (1.1) are satisfied. For the monodisperse laminar spray considered here, the liquid phase can be treated as a continuum, as indicated above, a convenient approximation for the analytical work attempted below. The variables describing the liquid phase are the droplet radius $a^{\prime}$, droplet number density $n^{\prime}$ and droplet axial and radial velocity components $u_{l}^{\prime}$ and $v_{l}^{\prime}$, in this continuum description that applies in the limit $R \gg l_{d}$, while the gas phase is characterized by its density and temperature $\rho^{\prime}$ and $T^{\prime}$, velocity components $u^{\prime}$ and $v^{\prime}$ and fuel mass fraction $Y$.

In the formulation, we shall further assume that the jet Reynolds number $R e_{j}=\rho_{j} U_{j} R / \mu$, where $\rho_{j}$ is the gas density at the jet exit and $\mu$ is the viscosity of the gas mixture, is large compared with unity for the flow to be slender, and yet not so large to ensure that the motion remains laminar and steady. In that case, the boundary-layer form of the axisymmetric conservation equations suffices to describe, with relative errors of order $R e_{j}^{-2}$, the resulting slender flow solution in terms of the axial and radial coordinates $x^{\prime}$ and $r^{\prime}$. The description of the gas phase reduces to the integration of the conservation equations for mass, momentum, species and energy. These equations, supplemented with the near-isobaric form of the equation of state, are to be integrated with initial conditions at $x^{\prime}=0$, corresponding to the spray and coflow properties at the exit plane, and boundary conditions at $r^{\prime}=0$ and as $r^{\prime} \rightarrow \infty$ for $x^{\prime}>0$. Numerical integrations of the corresponding parabolic problem for a selected number of cases were reported in Kim \& Chiu (1983).

To close the formulation, appropriate expressions must be selected for the source terms, which include the vaporization rate of each individual droplet $\dot{m}$ and the force $\left(f_{x}, f_{y}\right)$ acting on each droplet, as a consequence of its motion relative to the local surrounding gas. For simplicity, a low Reynolds number is assumed for the flow around the droplets, which implies that values of the relative velocity $u_{l}^{\prime}-u^{\prime}$ and $v_{l}^{\prime}-v^{\prime}$ are small compared with $\mu /\left(\rho^{\prime} a^{\prime}\right)$. Under those conditions, Stokes law

$$
\left(f_{x}, f_{y}\right)=6 \pi \mu a^{\prime}\left(u^{\prime}-u_{l}^{\prime}, v^{\prime}-v_{l}^{\prime}\right)
$$

can be used for the force acting on each droplet. On the other hand, with constant conductivity $k$ and specific heat at constant pressure $c_{p}$ assumed for the gas phase, the droplet mass vaporization rate reduces to (Godsave 1953; Liñán 1985)

$$
\dot{m}=4 \pi a^{\prime}\left(k / c_{p}\right) \ln \left(1+\frac{c_{p}\left(T^{\prime}-T_{B}^{\prime}\right)}{L_{v}}\right),
$$

which is a function of the local values of the gas temperature $T^{\prime}$ and droplet radius $a^{\prime}$. In writing (2.3), it has been taken into account that the droplets are initially in saturated equilibrium, at a uniform temperature near the boiling value such that no heat up is required prior to vaporization. Spalding numbers $c_{p}\left(T_{c}^{\prime}-T_{B}^{\prime}\right) / L_{v}$ of order 
unity are obtained for the characteristic values of the coflow temperature $T_{c}^{\prime}$ selected below, thereby giving typical values of the vaporization rate of order $4 \pi a_{j} k / c_{p}$.

\section{Characteristic time scales and controlling parameters}

An order-of-magnitude analysis of the different competing physical phenomena leads to useful estimates for the three characteristic times that are involved in the spray vaporization process. Thus, comparing convection and transverse diffusion in the gas-phase conservation equations leads to

$$
t_{d}=R^{2} / D_{T_{j}}
$$

as an estimate for the diffusion time across the jet, with $D_{T_{j}}=k /\left(\rho_{j} c_{p}\right)$ representing the thermal diffusivity of the gas at the spray exit. This time is equal to the residence time in the region of jet development, and typically differs from the droplet lifetime

$$
t_{a}=\frac{\rho_{l} a_{j}^{2}}{3 \rho_{j} D_{T_{j}}},
$$

obtained by dividing the initial droplet mass $(4 \pi / 3) a_{j}^{3} \rho_{l}$, where $\rho_{l}$ denotes the liquid fuel density, by the characteristic value of the vaporization rate $4 \pi a_{j}\left(k / c_{p}\right)$, obtained from (2.3) with a unity factor replacing the logarithmic term, as is appropriate when the Spalding number is of order unity. Note that, except for an irrelevant factor $2 /(3 P r)$, where $\operatorname{Pr}$ is the Prandtl number of the gas phase, the same estimate (3.2) is obtained for the characteristic time of droplet acceleration, as can be seen by equating the orders of magnitude of droplet acceleration $\rho_{l}(4 / 3) \pi a_{j}^{3} U_{j} / t_{a}$ and the characteristic value of the drag force $6 \pi \mu a_{j} U_{j}$, obtained from (2.2).

The time scale given in (3.2) characterizes the vaporization of each individual droplet. The collective effect of spray vaporization on the density, velocity, temperature and fuel-vapour evolution in the jet is however measured by a different time scale, a spray-interaction time,

$$
t_{s}=\frac{1}{4 \pi a_{j} n_{j} D_{T_{j}}}
$$

obtained as the ratio of the characteristic jet density $\rho_{j}$ to the volume rate of mass production through vaporization $4 \pi a_{j} n_{j} k / c_{p}$ (fuel mass per unit volume per unit time), the latter being the product of the characteristic value of the vaporization rate $4 \pi a_{j} k / c_{p}$ and the initial number of droplets per unit volume $n_{j}$. The scale given in (3.3) therefore corresponds to the characteristic time required for droplet vaporization to change appreciably - i.e. by a relative amount of order unity - the value of the gas density in the jet, as can also be obtained by comparing the convective term with the droplet source term in the gas-phase continuity equation. Similarly, the comparison of the convective and vaporization terms in the momentum, energy and fuel conservation equations also yields (3.3) as the characteristic time required for droplet vaporization to change significantly the values of the gas velocity, temperature and fuel mass fraction in the jet, respectively.

The two primary parameters that control the spray solution are obtained as the ratios of the above characteristic times. The first relevant parameter is the mass of liquid fuel per unit mass of gas in the spray stream,

$$
\lambda=\frac{(4 \pi / 3) a_{j}^{3} n_{j} \rho_{l}}{\rho_{j}},
$$


which is also equal to the characteristic time ratio $\lambda=t_{a} / t_{s}$. This parameter, measuring the dilution of the spray, will be taken as an order unity magnitude in the following development, as corresponds to spray configurations with characteristic values of the average distance between neighbouring droplets $l_{d}=n_{j}^{-1 / 3}$ of order $l_{d} \sim\left(\rho_{l} / \rho_{j}\right)^{1 / 3} a_{j} \sim 10 a_{j}$. Separate consideration will be given to the limiting cases $\lambda \gg 1$ and $\lambda \ll 1$, the latter being of interest in combustion applications, where small values of $\lambda$ of the order of the stoichiometric mixture fraction are often encountered. Note that in the limit $\lambda \gg 1$ of relatively dense sprays, the condition $l_{d} \gg a_{j}$ introduces an upper limit $\lambda \ll \rho_{l} / \rho_{j}$, so that the spray remains sufficiently dilute for the formulation to hold.

The second controlling parameter is the ratio of the characteristic time of jet evolution due to spray vaporization $t_{s}$ to the diffusion time $t_{d}$ :

$$
\varepsilon=\frac{t_{s}}{t_{d}}=\frac{1}{4 \pi a_{j} n_{j} R^{2}} .
$$

In terms of the three characteristic scales involved in (1.1), this ratio can be seen to be of order

$$
\varepsilon \sim\left(\frac{l_{d}}{R}\right)^{2}\left(\frac{l_{d}}{a_{j}}\right)
$$

With $l_{d} / a_{j}$ typically being a moderately large quantity of order $\left(\rho_{l} / \rho_{j}\right)^{1 / 3} \simeq 10$ for $\lambda \sim O(1)$, the resulting value of $\varepsilon$ depends on $l_{d} / R$. Small values of $\varepsilon$ are expected to appear in general in connection with the vaporization of sprays with multiple droplets, that is, sufficiently small values of $l_{d} / R$. On the other hand, in view of (3.6), it is clear that values of $\varepsilon$ of order unity or larger will be found only under conditions of extreme dilution, not often encountered in applications. Therefore, because of its expected wide range of applicability, the development of a deeper understanding of the limit $\varepsilon \ll 1$ is clearly worthwhile.

The parameter $\varepsilon$ was used previously as a small quantity for the asymptotic analysis of droplet cloud vaporization (Correa \& Sichel 1982a) and, as discussed in Correa \& Sichel $(1982 b)$, controls the group combustion characteristics in reactive configurations. Similar parameters were employed in other early theoretical studies to characterize these processes. Thus, as noted by Sichel \& Palaniswamy (1984), the parameter $\varepsilon$ is exactly equal to the inverse of the square of the Thiele modulus $\Psi$ employed by Labowsky \& Rosner (1978). Also, under the condition of small droplet Reynolds number used in deriving (2.3), $\varepsilon$ becomes equal to the reciprocal of the group combustion number $G$ introduced by Chiu and co-workers (Chiu \& Liu 1977; Chiu et al. 1978) times the Lewis number. In many practical applications, the parameter $\varepsilon$ takes on small values, causing vaporization to occur in a sheath or vaporization front that separates the spray, in saturated equilibrium, from the surrounding droplet-free hot gas, with the flame standing outside the spray in combustion configurations. This limit of sheath vaporization, analysed by Correa \& Sichel (1982a) for a spherical droplet cloud, will be investigated here for the axisymmetric vaporizing jet spray.

\section{Dimensionless formulation}

To non-dimensionalize the problem, the characteristic diffusion time $t_{d}$ will be used to construct scales for the streamwise length, $U_{j} t_{d}$, and for the gas and droplet radial velocities, $R / t_{d}=D_{T_{j}} / R$. Furthermore, the radial distance will be scaled with $R$, whereas the droplet and gas axial velocity components, the droplet radius and 
number density, and the gas temperature and density will be scaled with their values at the spray exit. With these scales, the complete set of dimensionless variables is given by $x=x^{\prime} /\left(U_{j} t_{d}\right), r=r^{\prime} / R, u=u^{\prime} / U_{j}, u_{l}=u_{l}^{\prime} / U_{j}, v=v^{\prime} /\left(R / t_{d}\right), v_{l}=v_{l}^{\prime} /\left(R / t_{d}\right)$, $a=a^{\prime} / a_{j}, n=n^{\prime} / n_{j}, T=T^{\prime} / T_{j}^{\prime}$ and $\rho=\rho^{\prime} / \rho_{j}$. The corresponding dimensionless form of the gas-phase equations is

$$
\begin{gathered}
\frac{\partial}{\partial x}(\rho u)+\frac{1}{r} \frac{\partial}{\partial r}(\rho r v)=\frac{n a}{\varepsilon} \ln \left[1+\frac{(T-1)}{\beta}\right] \\
\frac{\partial}{\partial x}\left(\rho u^{2}\right)+\frac{1}{r} \frac{\partial}{\partial r}(\rho r v u)-\frac{P r}{r} \frac{\partial}{\partial r}\left(r \frac{\partial u}{\partial r}\right) \\
=\frac{n a u_{l}}{\varepsilon} \ln \left[1+\frac{(T-1)}{\beta}\right]+\frac{3}{2} \frac{P r}{\varepsilon}\left(u_{l}-u\right) n a, \\
\frac{\partial}{\partial x}(\rho u T)+\frac{1}{r} \frac{\partial}{\partial r}(\rho r v T)-\frac{1}{r} \frac{\partial}{\partial r}\left(r \frac{\partial T}{\partial r}\right)=-\frac{n a}{\varepsilon}(\beta-1) \ln \left[1+\frac{(T-1)}{\beta}\right], \\
\frac{\partial}{\partial x}(\rho u Y)+\frac{1}{r} \frac{\partial}{\partial r}(\rho r v Y)-\frac{1}{L r} \frac{\partial}{\partial r}\left(r \frac{\partial Y}{\partial r}\right)=\frac{n a}{\varepsilon} \ln \left[1+\frac{(T-1)}{\beta}\right],
\end{gathered}
$$

while the equations for the liquid phase become

$$
\begin{aligned}
\frac{\partial}{\partial x}\left(n u_{l}\right)+\frac{1}{r} \frac{\partial}{\partial r}\left(n r v_{l}\right) & =0, \\
u_{l} \frac{\partial a^{3}}{\partial x}+v_{l} \frac{\partial a^{3}}{\partial r} & =-\frac{a}{\varepsilon \lambda} \ln \left[1+\frac{(T-1)}{\beta}\right], \\
u_{l} \frac{\partial u_{l}}{\partial x}+v_{l} \frac{\partial u_{l}}{\partial r} & =\frac{3 \operatorname{Pr}}{2 \varepsilon \lambda} \frac{u-u_{l}}{a^{2}}, \\
u_{l} \frac{\partial v_{l}}{\partial x}+v_{l} \frac{\partial v_{l}}{\partial r} & =\frac{3 \operatorname{Pr}}{2 \varepsilon \lambda} \frac{v-v_{l}}{a^{2}} .
\end{aligned}
$$

The problem is subject to the initial and boundary conditions

$$
x=0:\left\{\begin{array}{l}
r<1: \quad u=T=n=a=u_{l}=1, \quad Y=Y_{j}, \quad v_{l}=0, \\
r>1: \quad u=u_{c}, \quad T=T_{c}, \quad Y=0,
\end{array}\right.
$$

and

$$
x>0: \begin{cases}r=0: & v=\partial u / \partial r=\partial T / \partial r=\partial Y / \partial r=0, \\ r \rightarrow \infty: & u=u_{c}, \quad T=T_{c}, \quad Y=0 .\end{cases}
$$

The above equations must be supplemented with the dimensionless form of the equation of state. The description is simplified when changes in mean molecular weight of the gas mixture are neglected, thereby reducing the equation of state to

$$
\rho T=1 .
$$

We shall adopt this simplifying approximation in the following description. Nevertheless, quantitative departures, arising from variations in mean molecular weight in vaporization processes of typical liquid fuels, are worth investigating in the future.

Sources of mass, momentum and energy appear in the above equations associated with the drag force acting on the droplets and their vaporization rate, as given by (2.2) and (2.3). Linear combinations can be used to derive source-free equations. 
For instance, using (4.2) and (4.5)-(4.7) provides the spray momentum equation

$$
\frac{\partial}{\partial x}\left(\rho u^{2}+\lambda n a^{3} u_{l}^{2}\right)+\frac{1}{r} \frac{\partial}{\partial r}\left(\rho r v u+\lambda n a^{3} r v_{l} u_{l}\right)-\frac{\operatorname{Pr}}{r} \frac{\partial}{\partial r}\left(r \frac{\partial u}{\partial r}\right)=0,
$$

which can be integrated radially across the jet with the boundary conditions indicated above to provide the integral constraint,

$$
\int_{0}^{\infty} r\left[\rho u\left(u-u_{c}\right)+\lambda n a^{3} u_{l}\left(u_{l}-u_{c}\right)\right] \mathrm{d} r=\left(1-u_{c}\right)(\lambda+1) / 2,
$$

associated with the conservation of momentum flux. Source-free spray conservation equations can also be derived for energy and fuel mass, leading to two additional integral constraints that were used, together with (4.13), in monitoring the accuracy of the numerical integrations of (4.1)-(4.11).

As can be seen, besides $\varepsilon, \lambda$ and the Prandtl and Lewis numbers, $\operatorname{Pr}=\mu c_{p} / k$ and $L=k /\left(\rho_{j} c_{p} D_{T_{j}}\right)$, respectively, there exist three additional dimensionless parameters in the formulation, namely the dimensionless latent heat of vaporization $\beta=L_{v} /\left(c_{p} T_{B}^{\prime}\right)$ and the coflow to spray temperature and velocity ratios $T_{c}=T_{c}^{\prime} / T_{B}^{\prime}$ and $u_{c}=U_{c} / U_{j}$. The value $\beta=0.36$, corresponding to octane (Correa \& Sichel 1982a), is employed for the latent heat of vaporization in the computations below, which consider different values of $T_{c}-1$ and $u_{c}$. The computations include, in particular, jets discharging into a stagnant atmosphere $\left(u_{c}=0\right)$ and also coflow velocities equal to the spray velocity $\left(u_{c}=1\right)$, the latter being particularly simple, in that the solution for the axial velocity components reduces to $u=u_{l}=1$ everywhere in the flow field, thereby facilitating the computations.

It is worth mentioning that the formulation given above could be easily modified to be employed for the description of combustion of a fuel spray by a coflowing air stream. If the chemical reaction between the fuel vapour and the oxygen of the air is assumed to be infinitely fast, the description can be facilitated by incorporating chemically passive scalars to describe the composition and temperature, including in particular a mixture fraction $Z$ following a conservation equation identical to (4.4). In this limit of infinitely fast reaction, the flame lies where $Z$ is equal to its stoichiometric value $Z_{s}$, which enters as an additional parameter. The temperature peaks at the flame, which provides in this case the necessary heat source for group evaporation of the fuel spray. Studies of fuel-spray group combustion based on modifications of the above formulation are clearly worth pursuing.

\section{Sample numerical results}

The numerical scheme used to integrate (4.1)-(4.11) is second-order accurate, with an implicit marching procedure considered for the gas-phase equations, account being taken of the sources and sinks that appear in the conservation equations (4.1)-(4.4). Since $\rho T=1$, the solution for the temperature field becomes independent of the composition. The liquid phase is treated as a continuum, hence it can be discretized, defining a finite number of droplets, the outermost droplet of which defines the liquid-phase boundary, beyond which $n=0$.

The governing equations for the gas phase, including the source terms, are integrated with a second-order implicit finite-difference scheme, similar to that proposed by Tanehill, Anderson \& Pletcher (1984). The liquid-phase equations are written making use of the Lagrangian description; thus, the system (4.6)-(4.8) of partial differential equations is reduced to a system of ordinary differential equations, with an additional 


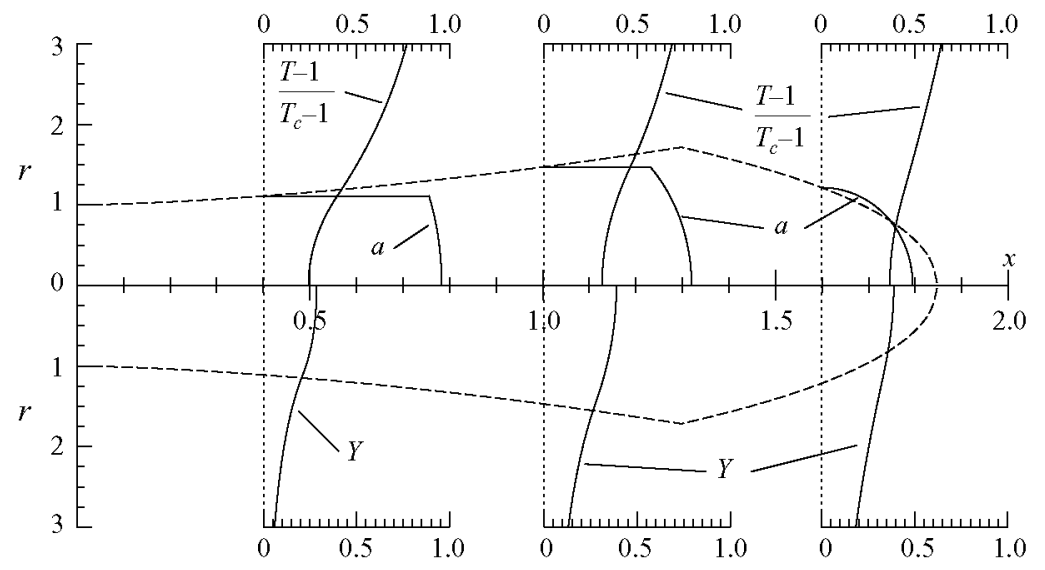

Figure 2. Profiles of temperature, droplet radius and fuel mass fraction across the vaporizing jet as obtained by integration of (4.1)-(4.11) for $u_{c}=0, L=1, Y_{j}=0.2, \operatorname{Pr}=0.7, \beta=0.36$, $T_{c}=2.15, \lambda=1$ and $\varepsilon=1$; the dashed line indicates the outer boundary of the spray.

differential equation which determines the radial position of each droplet as a function of its corresponding radial velocity. The gas-phase properties at each droplet position, needed to evaluate the source terms in (4.6)-(4.8), are obtained by linear interpolation. Similarly, the source terms are distributed to the neighbouring gas-phase mesh points by linear approximation. Therefore, the method used for the numerical integrations is very similar to that proposed by Aggarwal, Fix, \& Sirignano (1985) and Dukowicz (1980). However, in order to avoid numerical errors resulting from the stiffness of the source terms in (4.6)-(4.8) when $\varepsilon \ll 1$, the implicit trapezoidal rule is preferred and was employed for the integration of the differential equations of the liquid phase.

The integration of the droplet-density equation (4.5) was carried out by the finitevolume method with a cell-vertex scheme having dual control volumes (Blazek 2001). The vertices of the mesh at a given axial position are defined by each droplet radial position. Once the droplet axial velocity, radial velocity and corresponding radial position are calculated, the fluxes at each cell face are determined, providing the downstream evolution of the droplet density number.

Figures 2-6 correspond to numerical integrations of (4.1)-(4.11) for $L=1, \operatorname{Pr}=0.7$, $\beta=0.36, T_{c}=2.15, Y_{j}=0.2, u_{c}=0$, and different values of $\lambda$ and $\varepsilon$, including dilute $(\lambda=0.1)$ and dense $(\lambda=20)$ sprays. Results obtained in the sheath-vaporization limit $\varepsilon=0$, to be discussed later, are also provided in figures 3-6.

Figure 2 shows profiles of temperature, droplet radius and fuel mass fraction across the jet spray at three different axial locations. As can be seen, for the case $\varepsilon=1$ vaporization occurs in a distributed manner. In particular, although the vaporization is more pronounced at the edge of the spray, non-negligible vaporization of the droplets located along the axis can be noticed already at $x=0.4$. As a result, the fuel mass fraction increases from its initial value $Y_{j}=0.2$, giving profiles that peak at the axis. Also of interest is that heat transfer from the hot coflow increases the temperature within the spray to values significantly larger than the boiling temperature $T=1$.

Also shown in figure 2 is the outer boundary of the spray, which coincides initially with the outermost droplet trajectory. The radius of this boundary droplet decreases, however, downstream from the injector rim, as the droplet vaporizes in contact with the high-temperature coflow. This droplet is completely consumed at a finite distance from the injector $x \simeq 1.3$, so that farther downstream the spray boundary is defined as 

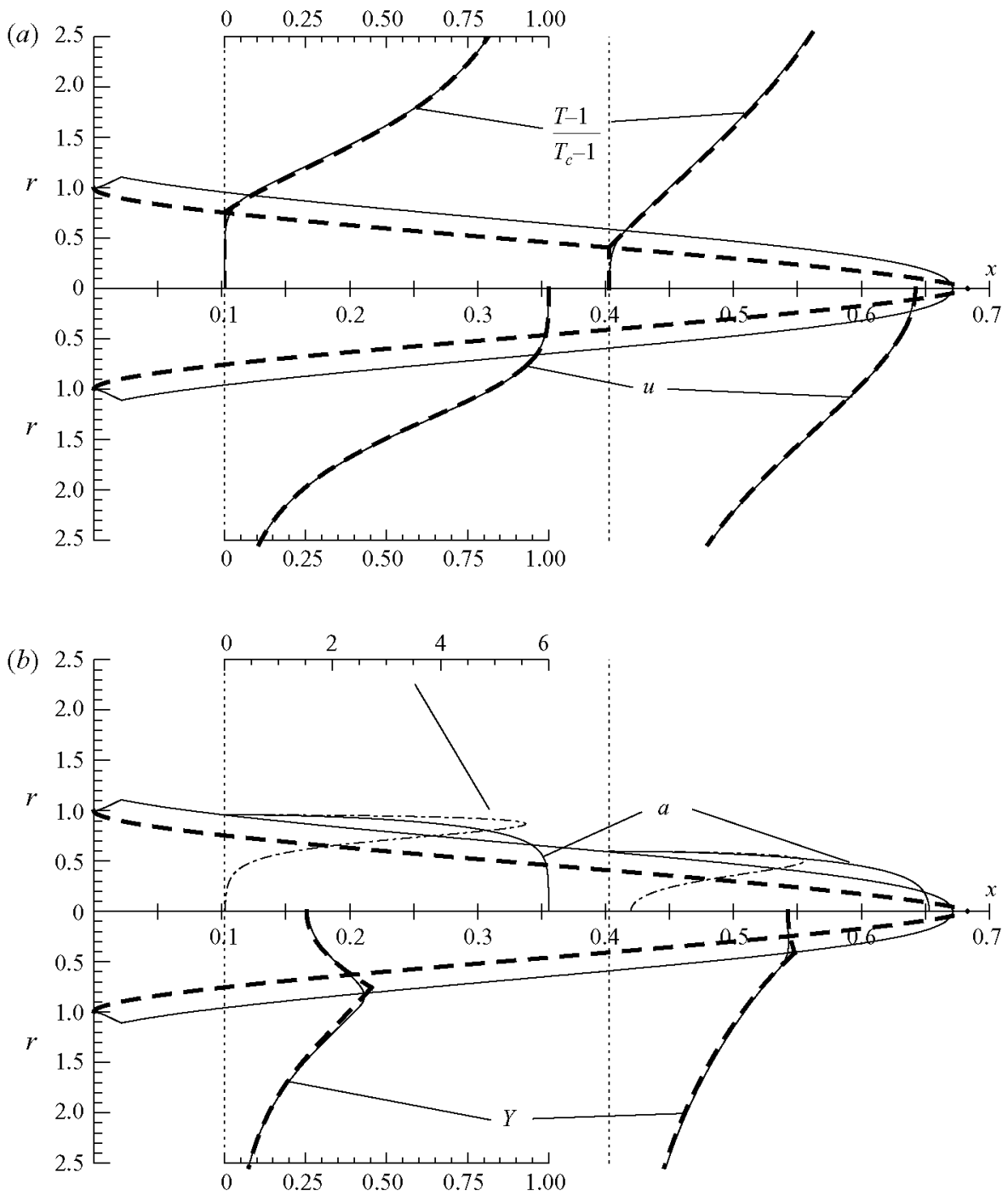

Figure 3. $(a, b)$ The vaporizing jet as obtained by integration of (4.1)-(4.11) for $u_{c}=0, L=1$, $Y_{j}=0.2, \operatorname{Pr}=0.7, \beta=0.36, T_{c}=2.15, \lambda=1$ and $\varepsilon=0.01$ (solid lines) along with results obtained in the sheath-vaporization limit $\varepsilon=0$ (dashed lines); the dot-dashed curves represent the radial profiles of the rescaled mass vaporization rate $n a \ln [1+(T-1) / \beta] / \varepsilon$. The scales are indicated for the profiles at the first axial location, with a different scale used for the mass vaporization rate.

the location where $a=0$, corresponding to vaporizing droplets located initially within the jet away from the injector edge.

The plots in 3-5 show profiles of temperature, fuel mass fraction, gas axial velocity, droplet radius and mass vaporization rate at two different axial locations. For all three cases considered, corresponding to relatively small values of $\varepsilon$, the solution shows a structure not present in figure 2. The distinct flow structure that emerges includes a thin vaporization layer, where the vaporization rate is concentrated and the fuel-vapour mass fraction reaches its peak value, separating an outer non-vaporizing region with $a=0$ from an inner equilibrium region, where the temperature, velocity 

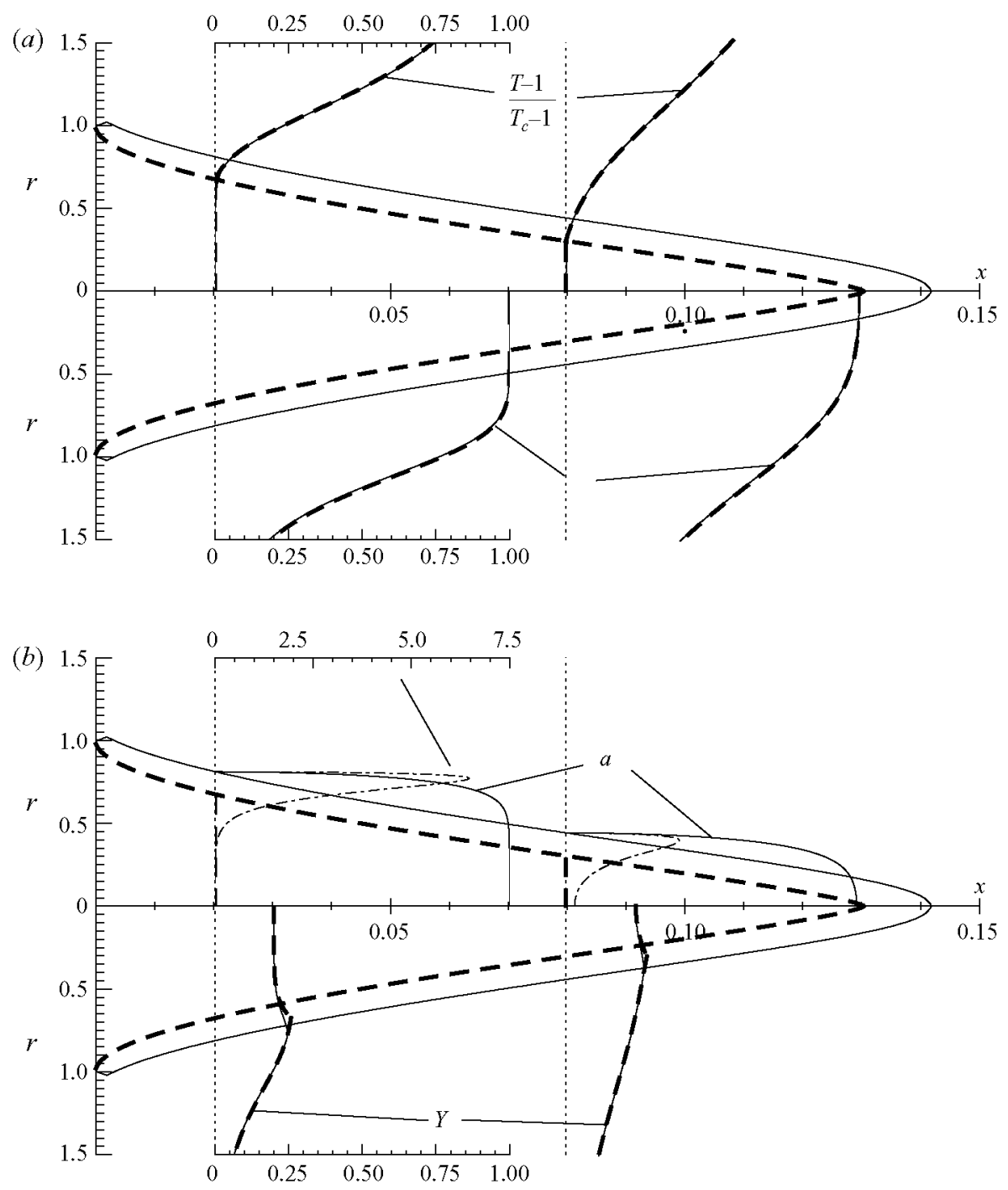

FIgURE 4. $(a, b)$ The vaporizing jet as obtained by integration of (4.1)-(4.11) for $u_{c}=0, L=1$, $Y_{j}=0.2, \operatorname{Pr}=0.7, \beta=0.36, T_{c}=2.15, \lambda=0.1$ and $\varepsilon=0.01$ (solid lines) along with results obtained in the sheath-vaporization limit $\varepsilon=0$ (dashed lines); the dot-dashed curves represent the radial profiles of the rescaled mass vaporization rate $n a \ln [1+(T-1) / \beta] / \varepsilon$. The scales are indicated for the profiles at the first axial location, with a different scale used for the mass vaporization rate.

and droplet radius remain approximately equal to their injector values $T=u=a=1$. This sheath-vaporization regime, identified by Correa \& Sichel (1982a) when dealing with the vaporization of a spherical fuel-droplet cloud, will be further considered in the following section for the analysis of the jet structure.

Figures 3-5 also show the outer boundary of the spray, which increases with $\lambda$, as may be seen from the different $x$ scales in the figures. The evolution of the corresponding spray shape for decreasing values of $\varepsilon$ and two different coflow velocities is shown in figure 6 . The downstream distance for vaporization of the boundary droplet leaving the injector rim is proportional to the initial jet velocity $U_{j}$ 

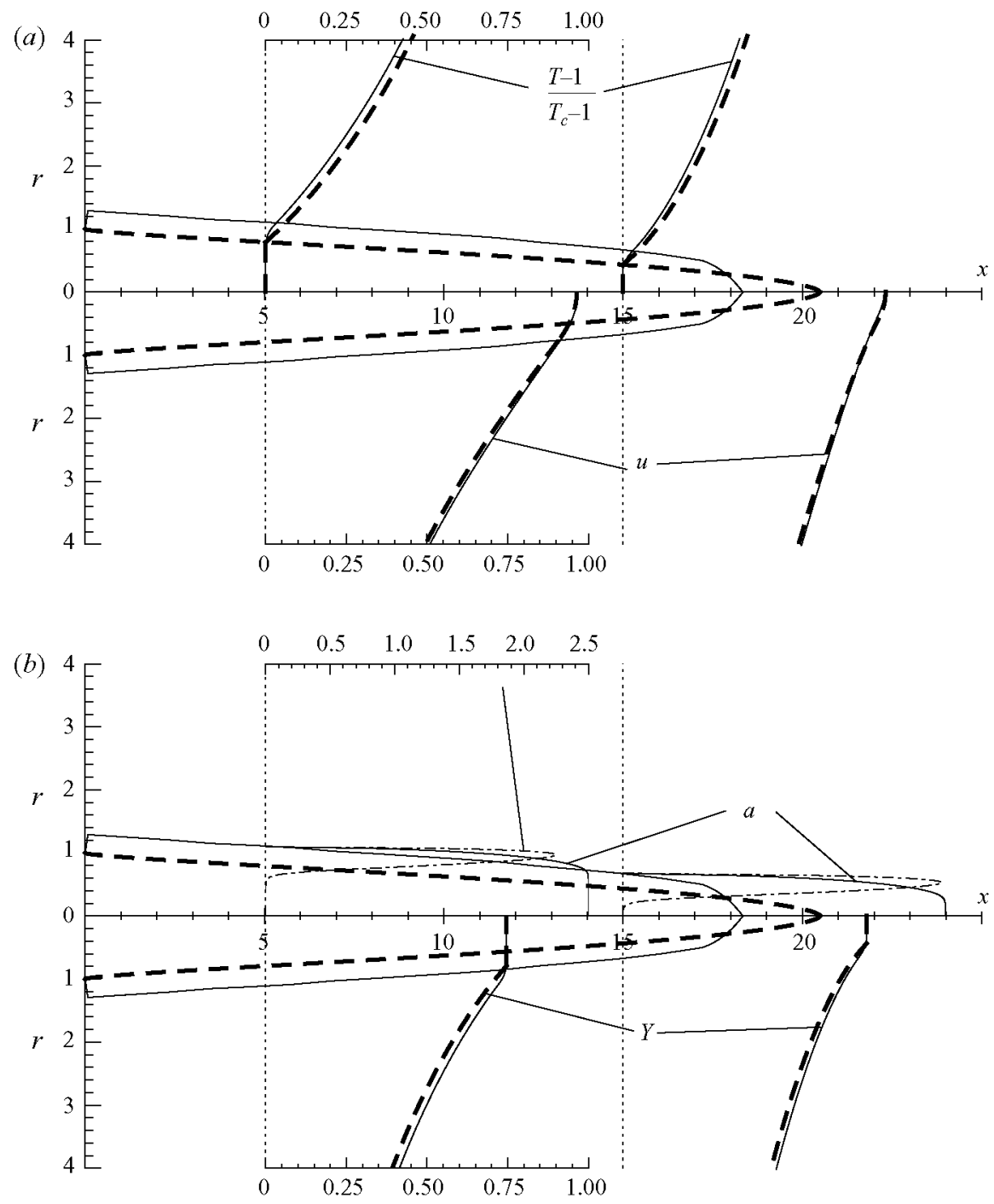

FiguRE 5. $(a, b)$ The vaporizing jet as obtained by integration of (4.1)-(4.11) for $u_{c}=0, L=1$, $Y_{j}=0.2, \operatorname{Pr}=0.7, \beta=0.36, T_{c}=2.15, \lambda=20$ and $\varepsilon=10^{-3}$ (solid lines) along with results obtained in the sheath-vaporization limit $\varepsilon=0$ (dashed lines); the dot-dashed curves represent the radial profiles of the rescaled mass vaporization rate $n a \ln [1+(T-1) / \beta] / \varepsilon$. The scales are indicated for the profiles at the first axial location, with a different scale used for the mass vaporization rate.

times the vaporization time of a single droplet $t_{a}$. With the scales selected here, this distance becomes proportional to $\varepsilon \lambda$ when expressed in dimensionless form, as can be inferred from (4.6). Therefore, as $\varepsilon$ decreases for a given value of $\lambda$, the corresponding vaporization distance for the boundary droplet also decreases, a result seen in the plots of figure 6 .

The downstream position where the droplet located initially at the axis vaporizes completely, which is the location where the boundary of the spray intersects the axis, defines the spray penetration distance $x_{v}$. This is seen in figures 3-6 to depend on the spray dilution through the liquid-to-gas spray mass ratio $\lambda$. Dilute sprays 

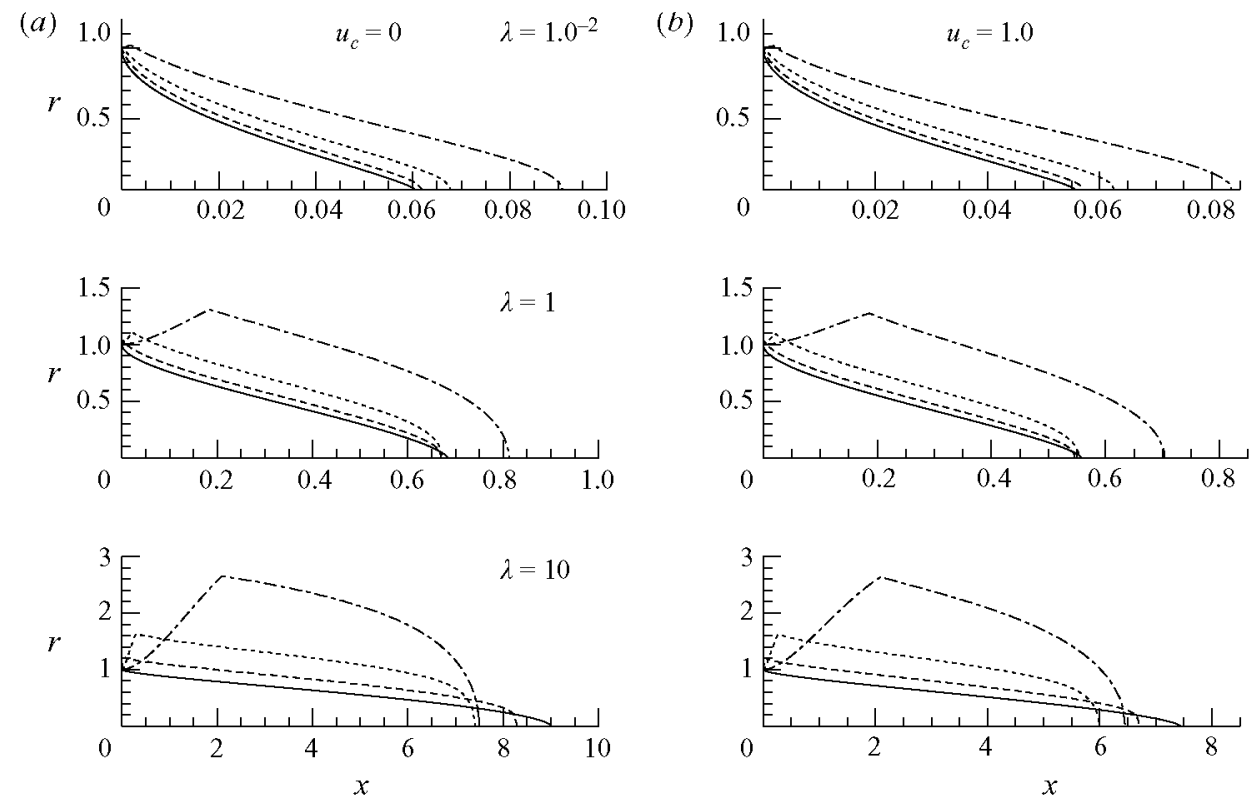

FIgURE 6. $(a, b)$ The boundary of the liquid phase where $a=0$ obtained by integration of (4.1)-(4.11) for $L=1, \operatorname{Pr}=0.7, \beta=0.36$ and $T_{c}=2.15$ for three different values of $\lambda$ and for $\varepsilon=10^{-1}$ (dash-dotted line), $\varepsilon=10^{-2}$ (dotted line) and $\varepsilon=10^{-3}$ (dashed line). The solid line represents the vaporization-layer location $r_{v}(x)$ obtained in the limit $\varepsilon=0$.

corresponding to $\lambda \ll 1$ vaporize at a short distance from the exit plane, whereas dense sprays with $\lambda \gg 1$ penetrate farther. The rough estimate

$$
x_{v}=\frac{\lambda}{2\left(T_{c}-1\right) / \beta}
$$

for the dependence of $x_{v}$ on $\lambda$ follows from equating the total heat provided by the coflow per unit time, which can be estimated as the product of the characteristic radial heat flux $k\left(T_{c}^{\prime}-T_{B}^{\prime}\right) / R$ and the spray lateral surface $2 \pi R x_{v}^{\prime}$, to the amount of heat needed per unit time to vaporize the droplets, obtained as the product of the liquid mass flow rate $\pi R^{2} U_{j} n_{j}(4 / 3) \pi a_{j}^{3} \rho_{l}$ and the latent heat of vaporization $L_{v}$. As seen below, for very long and very short sprays, corresponding to the two limiting cases $\lambda \gg 1$ and $\lambda \ll 1$, the radial heat flux is modified, so that the analytical expressions that are obtained for the penetration distance in the sheath-vaporization limit $\varepsilon=0$, given later in (8.14) and (9.7), exhibit dependences on parameters that differ from those displayed in (5.1).

\section{The sheath-vaporization limit}

The appearance of the sheath-vaporization regime for small values of $\varepsilon$, clearly apparent in the numerical results shown in figures $3-5$, can be anticipated by observing that in the limit $\varepsilon \rightarrow 0$ the solution of (4.1)-(4.4) - or that of (4.6) - leads to $a \ln [1+(T-1) / \beta]=0$, indicating the existence of a thin vaporization front located at $r=r_{v}(x)$ separating an outer region for $r>r_{v}$ where no droplets are found $(a=0)$, and an inner region for $r<r_{v}$ where the temperature remains equal to the boiling temperature in the first approximation $(T=1)$. Droplets vaporize only within the thin 
vaporization layer, of characteristic thickness $\varepsilon^{1 / 2}$, which appears as a localized sink of energy and source of mass, causing the profiles of $T$ and $Y$ to show a discontinuous radial gradient at $r=r_{v}$, with $Y$ reaching its peak value $Y_{v}$ there. Since $T-1 \sim \varepsilon^{1 / 2}$ in the vaporization layer, with both $n$ and $a$ remaining of order unity, the resulting dimensionless mass vaporization rate $n a \ln [1+(T-1) / \beta] / \varepsilon$ becomes of order $\varepsilon^{-1 / 2}$, as can be observed in the plots of figures 3-5. Because of the concentrated mass release, the droplet and gas radial velocity components, $v_{l}$ and $v$, which are equal in the first approximation as can be seen from (4.8) in the limit $\varepsilon \rightarrow 0$, exhibit a jump across the vaporization layer. The axial velocity components $u$ and $u_{l}$ are also almost equal, as follows from (4.7) with $\varepsilon \ll 1$. Vaporization does not result in a net axial momentum exchange between the liquid and gas phases, so that the values of $u$ and $u_{l}$ and those of their radial gradients are equal on both sides of the vaporization front.

The leading-order asymptotic analysis in the limit $\varepsilon \rightarrow 0$ leads to a free-boundary problem in which $r_{v}(x)$ is to be determined as part of a nonlinear parabolic problem. In the notation employed, the flow properties at the vaporization front will be denoted by the subscript $v$, with the + and - signs used to refer to the outer and inner sides when, as occurs for instance with the radial velocity and with the temperature gradient, there is a leading-order change across the front caused by vaporization.

\subsection{The outer non-vaporizing streams}

As previously anticipated, for $r>r_{v}$, the solution of (4.6) in the limit $\varepsilon=0$ yields $a=0$, thereby reducing the solution for the gas phase to the integration of

$$
\begin{aligned}
\frac{\partial}{\partial x}(\rho u)+\frac{1}{r} \frac{\partial}{\partial r}(\rho r v) & =0, \\
\frac{\partial}{\partial x}\left(\rho u^{2}\right)+\frac{1}{r} \frac{\partial}{\partial r}(\rho r v u)-\frac{P r}{r} \frac{\partial}{\partial r}\left(r \frac{\partial u}{\partial r}\right) & =0, \\
\frac{\partial}{\partial x}(\rho u T)+\frac{1}{r} \frac{\partial}{\partial r}(\rho r v T)-\frac{1}{r} \frac{\partial}{\partial r}\left(r \frac{\partial T}{\partial r}\right) & =0, \\
\frac{\partial}{\partial x}(\rho u Y)+\frac{1}{r} \frac{\partial}{\partial r}(\rho r v Y)-\frac{1}{L r} \frac{\partial}{\partial r}\left(r \frac{\partial Y}{\partial r}\right) & =0 .
\end{aligned}
$$

For $r<r_{v}$, on the other hand, $T=1$ and, therefore, $\rho=1$ according to (4.11), so that (4.1) reduces to

$$
\frac{\partial u}{\partial x}+\frac{1}{r} \frac{\partial}{\partial r}(r v)=0 .
$$

In the absence of vaporization, the radius of each droplet remains unperturbed, as can be seen by inspection of (4.6), so that $a=1$ for $r<r_{v}$. Furthermore, observation of (4.7) and (4.8) indicates that $u-u_{l} \sim v-v_{l} \sim O(\varepsilon)$, so that in the first approximation one may use $u_{l}=u$ and $v_{l}=v$. When this condition is used along with (6.5) in (4.5), the equation $u_{l} \partial n / \partial x+v_{l} \partial n / \partial r=0$ is obtained, which yields $n=1$ for $r<r_{v}$ upon integration along the droplet trajectories. The small differences $u-u_{l} \sim \varepsilon$ are sufficiently large for the Stokes force to be non-negligible in (4.2) and (4.7). To avoid the presence of the resulting singular term, the leading-order results $T=1, a=1, n=1, u_{l}=u$ and $v_{l}=v$ are used in the spray momentum equation (4.12) to give the alternative equation

$$
u \frac{\partial u}{\partial x}+v \frac{\partial u}{\partial r}-\frac{P r}{1+\lambda} \frac{1}{r} \frac{\partial}{\partial r}\left(r \frac{\partial u}{\partial r}\right)=0,
$$


for the computation of $u=u_{l}$ for $r<r_{v}$. Finally, the fuel conservation equation reduces with $\rho=1$ to

$$
\frac{\partial}{\partial x}(u Y)+\frac{1}{r} \frac{\partial}{\partial r}(r v Y)-\frac{1}{L r} \frac{\partial}{\partial r}\left(r \frac{\partial Y}{\partial r}\right)=0,
$$

which completes the set of equations in the outer non-vaporizing streams.

\subsection{The vaporization layer}

The study of the self-similar inner structure of the vaporization layer provides a set of boundary conditions at $r=r_{v}$ to be used in integrating (6.1)-(6.7). Across this layer, of characteristic thickness $\varepsilon^{1 / 2}$, the values of $T, u$ and $Y$ only change by a small amount of order $\varepsilon^{1 / 2}$ from their order-unity values $T=1, u=u_{v}$ and $Y=Y_{v}$, respectively, whereas $v, a$ and $n$ experience changes of order unity. The relative velocity components $u-u_{l} \sim \varepsilon$ and $v-v_{l} \sim \varepsilon^{1 / 2}$ are sufficiently small for (4.7) and (4.8) to be replaced at leading order by $u=u_{l}$ and $v=v_{l}$. The solution can be determined by rewriting (4.1)-(4.6) in terms of the rescaled radial coordinate $\xi=\left(r-r_{v}\right) / \varepsilon^{1 / 2}$ and the rescaled variables $\theta=(T-1) / \varepsilon^{1 / 2}, U=\left(u-u_{v}\right) / \varepsilon^{1 / 2}$ and $\phi=\left(Y-Y_{v}\right) / \varepsilon^{1 / 2}$. In the formulation, the subscript $\xi$ denotes differentiation with respect to this variable.

The development begins by integrating once $-u_{v}\left(\mathrm{~d} r_{v} / \mathrm{d} x\right) n_{\xi}+(n v)_{\xi}=0$, corresponding to the reduced form of (4.5), with boundary conditions $n=1$ and $v=v_{-}$on the spray side (i.e. as $\xi \rightarrow-\infty$ ) to give

$$
n\left(v-u_{v} \frac{\mathrm{d} r_{v}}{\mathrm{~d} x}\right)=v_{-}-u_{v} \frac{\mathrm{d} r_{v}}{\mathrm{~d} x}
$$

Introduction of the rescaled variables into (4.1) and (4.6) gives

$$
\lambda\left(u_{v} \frac{\mathrm{d} r_{v}}{\mathrm{~d} x}-v_{-}\right)\left(a^{3}\right)_{\xi}=v_{\xi}=\frac{n a \theta}{\beta},
$$

where (6.8) has been employed to express the factor multiplying $\left(a^{3}\right)_{\xi}$ in a form independent of $\xi$. Equation (6.9) can be used in (4.3) and (4.4) to give

$$
\frac{\theta_{\xi \xi}}{\beta}=-\frac{\phi_{\xi \xi}}{L\left(1-Y_{v}\right)}=\frac{n a \theta}{\beta},
$$

and in (4.2) to give

$$
U_{\xi \xi}=0 .
$$

First integrations of (6.9), (6.10) and (6.11) with boundary conditions as $\xi \rightarrow-\infty$ give

$$
-\lambda\left(u_{v} \frac{\mathrm{d} r_{v}}{\mathrm{~d} x}-v_{-}\right)\left(1-a^{3}\right)=v-v_{-}=\frac{\theta_{\xi}}{\beta}=-\frac{\phi_{\xi}-\left(\phi_{\xi}\right)_{-}}{L\left(1-Y_{v}\right)}
$$

and $U_{\xi}=\left(U_{\xi}\right)_{-}$. Evaluating these expressions as $\xi \rightarrow+\infty$ provides the jump conditions across the vaporization layer

$$
-\lambda\left(u_{v} \frac{\mathrm{d} r_{v}}{\mathrm{~d} x}-v_{-}\right)=v_{+}-v_{-}=\frac{1}{\beta}\left(\frac{\partial T}{\partial r}\right)_{+}=-\frac{\left(\frac{\partial Y}{\partial r}\right)_{+}-\left(\frac{\partial Y}{\partial r}\right)_{-}}{L\left(1-Y_{v}\right)}
$$

and

$$
\left(\frac{\partial u}{\partial r}\right)_{+}=\left(\frac{\partial u}{\partial r}\right)_{-}
$$

which have been written in terms of the original spray variables. 
The jump conditions given above in (6.13) and (6.14) are needed for the integration of the outer equations given in (6.1)-(6.7). No additional details are necessary at the leading order considered here. Nevertheless, for completeness of the presentation, we give below the detailed solution for $n, a$ and $\theta$ across the vaporization layer.

The first equation in (6.12) can be employed together with (6.8) to write

$$
n=\frac{1}{1+\lambda\left(1-a^{3}\right)},
$$

which can be evaluated with $a=0$ to determine the value of the droplet number density on the outer side of the vaporization layer, a function of the dilution given by $n_{+}=(1+\lambda)^{-1}$. On the other hand, according to (6.10), the temperature variation across the vaporization layer can be computed from

$$
\theta_{\xi \xi}=n a \theta\left\{\begin{array}{l}
\theta(-\infty)=0, \\
\theta(+\infty)=q+\xi,
\end{array}\right.
$$

where the heat flux $q_{+}=(\partial T / \partial r)_{+}$is to be determined as part of the integration of the outer problem. To facilitate the computation, the expression for the temperature gradient $\theta_{\xi}=q_{+}\left(1-a^{3}\right)$, given in (6.12), can be used together with (6.15) to rewrite (6.16) in the form

$$
\theta \frac{\mathrm{d} \theta}{\mathrm{d} a}=-3 q_{+}^{2} a\left(1-a^{3}\right)\left[1+\lambda\left(1-a^{3}\right)\right],
$$

which leads to

$$
\theta / q_{+}=\sqrt{6}\left[\frac{3}{10}-\frac{a^{2}}{2}+\frac{a^{5}}{5}+\lambda\left(\frac{9}{40}-\frac{a^{2}}{2}-\frac{a^{8}}{8}+\frac{2 a^{5}}{5}\right)\right]^{1 / 2}
$$

upon integration with boundary condition $\theta=0$ when $a=1$. Evaluating (6.18) with $a=0$ provides $\theta_{0}=3 q_{+}[(4+3 \lambda) / 20]^{1 / 2}$ for the value of the temperature increase at the inner-layer location $\xi=\xi_{0}=3[(4+3 \lambda) / 20]^{1 / 2}$, where $a=0$. For $\xi>\xi_{0}$, the temperature is simply given by $\theta=q+\xi$, as follows from integrating (6.16) with $a=0$, whereas for $\xi<\xi_{0}$ the temperature is determined through (6.18) in terms of the droplet-radius distribution,

$$
\xi_{0}-\xi=\int_{0}^{a} \frac{3 a\left[1+\lambda\left(1-a^{3}\right)\right]}{\theta / q_{+}} \mathrm{d} a,
$$

obtained from $\theta_{\xi}=q_{+}\left(1-a^{3}\right)$, with the function (6.18) used to express the denominator in the above integral as a function of $a$.

\subsection{Mixing layer near the injector rim}

Initial conditions for the integration of (6.1)-(6.7) follow from investigating the near-injector region, an analysis presented below. For $x$ in the range $\lambda \varepsilon \ll x \ll 1$, the vaporization front has already developed, but remains embedded in the mixing layer that separates the jet and the coflow, whose thickness increases downstream from the injector rim proportional to the square root of the streamwise distance. The analysis of this region employes the local coordinate $\eta=(r-1) / \sqrt{x}$ and the mixture fraction $\psi=\sqrt{x} F(\eta)$, defined such that the rescaled front location is given by $\eta_{v}=\left(r_{v}-1\right) / \sqrt{x}$ while the velocity components can be expressed in the form $u=T F_{\eta}$ and $V=\sqrt{x} v=T\left(\eta F_{\eta}-F\right) / 2$, where the subscript $\eta$ indicates differentiation with respect to this similarity coordinate. Introducing these variables into (6.2) and (6.3) 

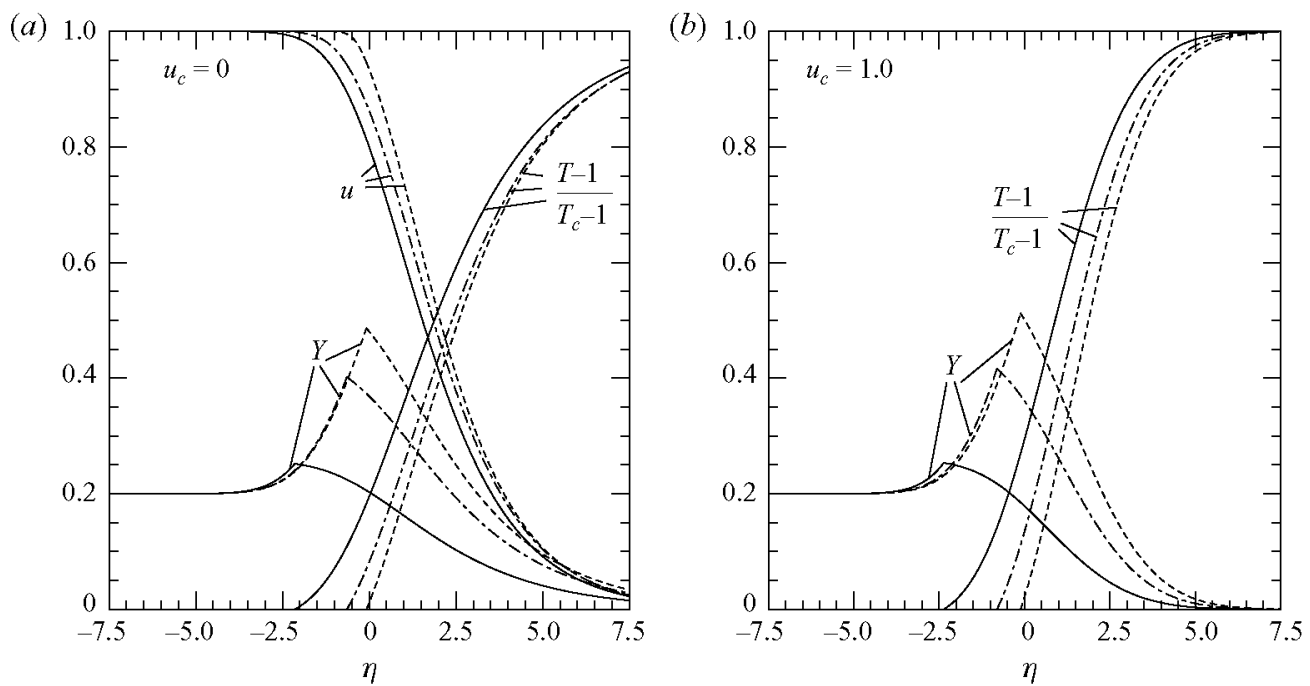

Figure 7. $(a, b)$ Profiles of temperature, fuel mass fraction and axial velocity in the mixing layer as obtained for $\beta=0.36, T_{c}=2.15, \operatorname{Pr}=0.7, L=1, Y_{j}=0.2$ and $\lambda=10^{-1}$ (solid line), $\lambda=1$ (dash-dotted line) and $\lambda=10$ (dashed line); with $u_{c}=1$ the solution for the axial velocity reduces to $u=1$ and is not shown in the figure.

gives

$$
\begin{aligned}
\left(T F_{\eta}\right)_{\eta \eta}+\frac{1}{2 P r} F\left(T F_{\eta}\right)_{\eta} & =0 \\
T_{\eta \eta}+\frac{1}{2} F T_{\eta} & =0
\end{aligned}
$$

whereas (6.6) yields

$$
F_{\eta \eta \eta}+\frac{1+\lambda}{2 \operatorname{Pr}} F F_{\eta \eta}=0,
$$

and the fuel mass fraction satisfies

$$
Y_{\eta \eta}+\frac{1}{2 L} F Y_{\eta}=0
$$

The solution involves integration of (6.20), (6.21) and (6.23) for $\eta>\eta_{v}$ with boundary conditions $F_{\eta}-u_{c} / T_{c}=T-T_{c}=Y=0$ as $\eta \rightarrow \infty$ and $T-1=F_{\eta}-u_{v}=Y-Y_{v}=0$ at $\eta=\eta_{v}$, and of (6.22) and (6.23) for $\eta<\eta_{v}$ with boundary conditions $F-\eta=Y-Y_{j}=0$ as $\eta \rightarrow-\infty$ and $F_{\eta}-u_{v}=Y-Y_{v}=0$ at $\eta=\eta_{v}$. The additional conditions

$$
\begin{gathered}
-\frac{\lambda}{2} F_{-}=\frac{1}{2}\left(F_{-}-F_{+}\right)=\frac{1}{\beta}\left(T_{\eta}\right)_{+}=\frac{\left(Y_{\eta}\right)_{-}-\left(Y_{\eta}\right)_{+}}{L\left(1-Y_{v}\right)}, \\
\left(T_{\eta} F_{\eta}+F_{\eta \eta}\right)_{+}-\left(F_{\eta \eta}\right)_{-}=0,
\end{gathered}
$$

at $\eta=\eta_{v}$, corresponding respectively to (6.13) and (6.14), serve to close the problem. For given values of $\beta, \lambda, T_{c}$ and $Y_{j}$, the integration provides the temperature, velocity and fuel mass fraction across the mixing layer, including the vaporization-layer values $u_{v}, Y_{v}$ and $\eta_{v}$. Sample profiles are shown in figure 7 for $u_{c}=0$ and $u_{c}=1$. The dependence of $\eta_{v}$ with $\lambda$ for different values of $T_{c}$ and $u_{c}$ is shown in figure 8 . As can be seen, the location of the vaporization layer depends on the value of $\lambda$. The two limiting cases of very dense and very dilute sprays are addressed below. 

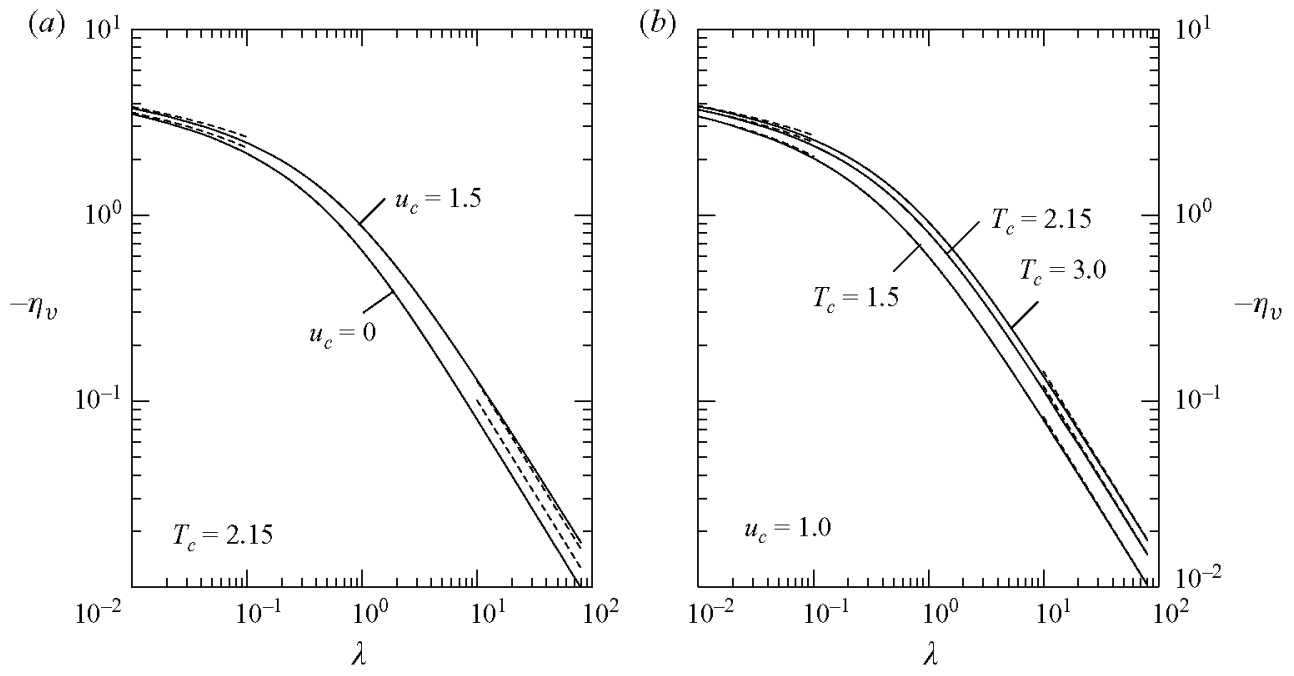

Figure 8 . $(a, b)$ The variation of $\eta_{v}$ as a function of $\lambda$ for $\beta=0.36, \operatorname{Pr}=0.7$, and different values of $T_{c}$ and $u_{c}$. The dashed lines represent the asymptotic behaviours for $\lambda \ll 1$ and $\lambda \gg 1$.

\section{Sheath-vaporization results}

The solution for the jet in the sheath-vaporization regime can be determined by integration of (6.1)-(6.4) with boundary conditions $u-u_{c}=T-T_{c}=Y=0$ as $r \rightarrow \infty$ and $u-u_{v}=T-1=Y-Y_{v}=0$ as $r \rightarrow r_{v}$ and of (6.5)-(6.7) with boundary conditions $v=\partial u / \partial r=\partial Y / \partial r=0$ at $r=0$ and $u-u_{v}=Y-Y_{v}=0$ as $r \rightarrow r_{v}$. Initial conditions correspond to the self-similar solutions identified above at $x \ll 1$. The two problems are coupled through the additional constraints (6.13) and (6.14). The solution determines in particular the boundary values $u_{v}(x), Y_{v}(x), v_{+}(x)$ and $v_{-}(x)$ along with the evolution of the vaporization front $r_{v}(x)$ from its initial location $r_{v}(0)=1$.

Figures 3-5 show by dashed curves the profiles of temperature, axial velocity, droplet radius and fuel mass fraction determined in the sheath-vaporization limit. As can be seen, the agreement with the results of numerical integrations of the original spray equations for small values of $\varepsilon$ is excellent. The location of the vaporization front $r_{v}(x)$ is also shown in these figures, and also in figure 6 , where it can be clearly seen that the spray boundary computed for decreasing values of $\varepsilon$ approaches the vaporization front of the sheath-vaporization limit, with departures appearing at small distances $x \sim \varepsilon \lambda$, in the initial region where the vaporization front is forming.

Of particular interest in applications is the downstream distance of spray penetration $x_{v}^{\prime}$ before complete vaporization is achieved. In the sheath-vaporization limit, this penetration distance corresponds to the downstream location $x_{v}$ at which the vaporization front $r_{v}(x)$ reaches the axis, i.e. $r_{v}\left(x_{v}\right)=0$. The variation of this distance with $\lambda$ is compared in figure 9 with results of numerical integrations of the original problem (4.1)-(4.11) for three values of $\varepsilon$ and two different coflow velocities. As expected, the sheath-vaporization limit correctly predicts the penetration distance of sprays with $\varepsilon \ll 1$, with relative errors being typically small (e.g. of the order of $20 \%$ for $\lambda \sim O(1)$ and $\varepsilon=0.1)$. Values of $x_{v}$ obtained with the sheath-vaporization reduced problem for different coflow conditions are shown in figure 10, where the 

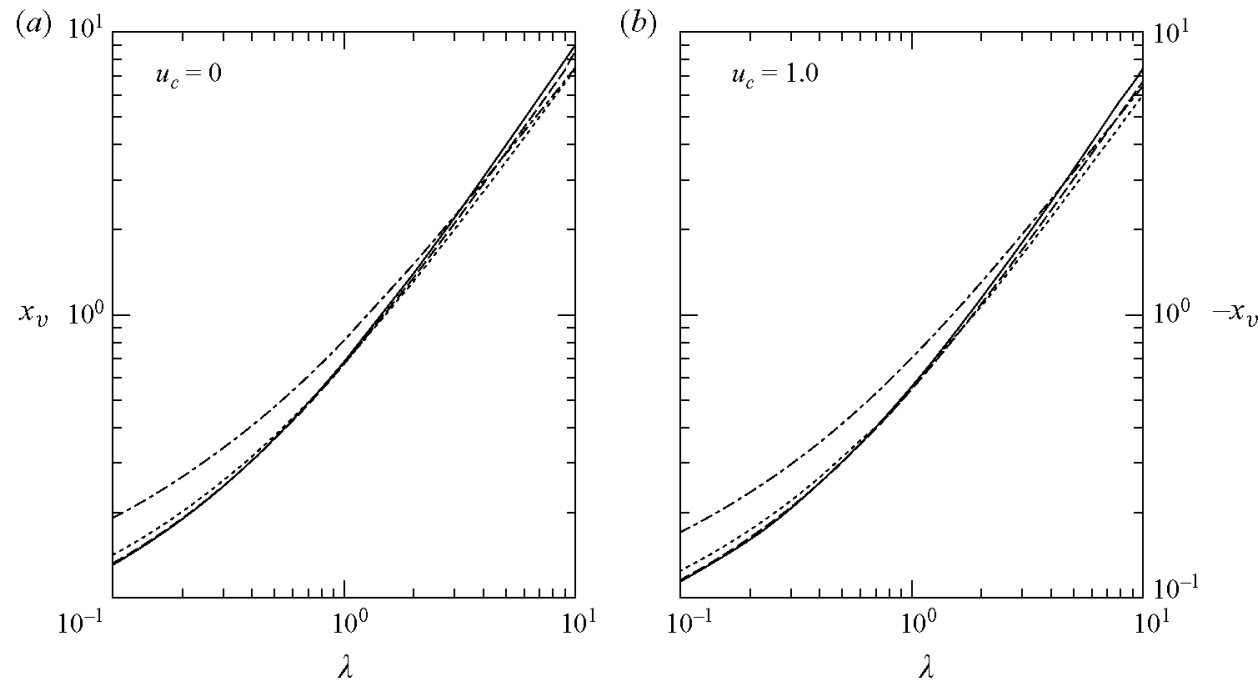

FIgURE 9. $(a, b)$ The spray penetration distance obtained with $T_{c}=2.15, \beta=0.36$ and $\operatorname{Pr}=0.7$ by integration of (4.1)-(4.11) for $\varepsilon=10^{-1}$ (dash-dotted line), $\varepsilon=10^{-2}$ (dotted line) and $\varepsilon=10^{-3}$ (dashed line) compared with the vaporization distance obtained in the limit $\varepsilon \rightarrow 0$ (solid line).
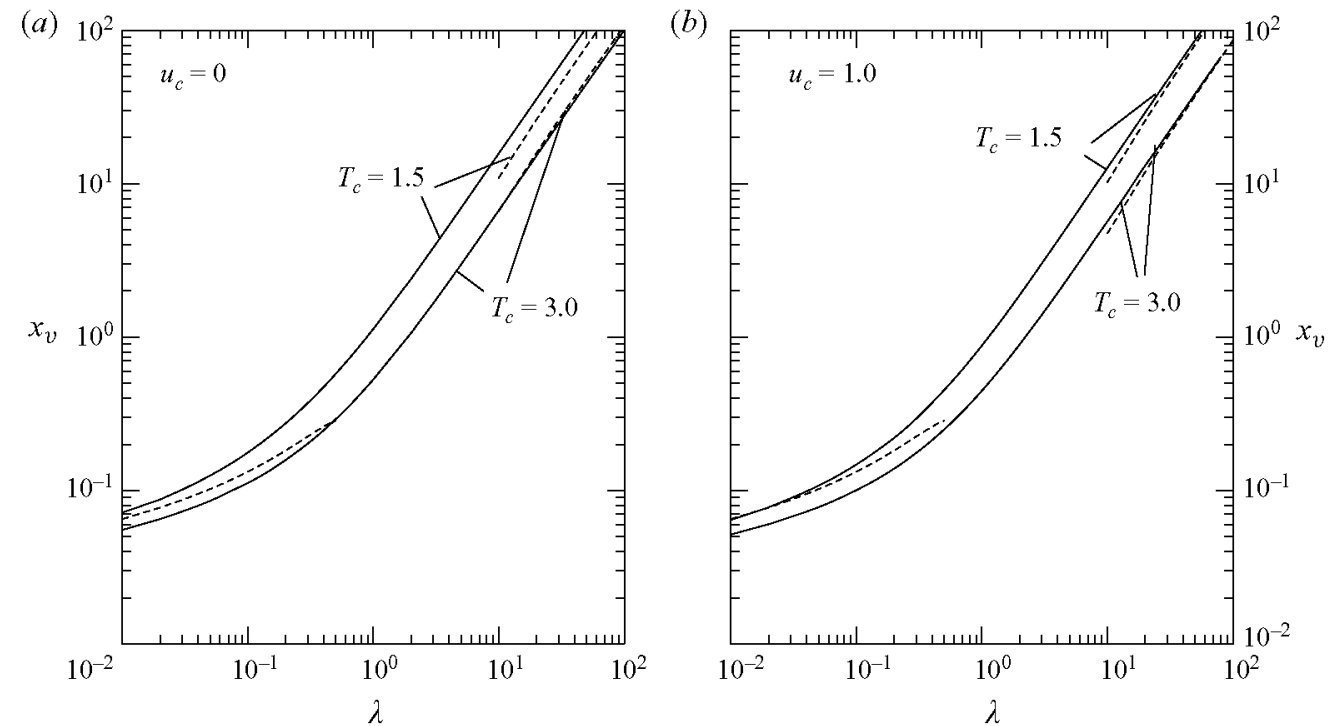

Figure 10. $(a, b)$ The spray penetration distance $x_{v}$ obtained in the limit $\varepsilon \rightarrow 0$ with $\beta=0.36$ and $P r=0.7$ for two different values of $T_{c}$ and $u_{c}$. The asymptotic leading-order predictions given for $\lambda \ll 1$ in (9.7) and for $\lambda \gg 1$ in (8.14) are also plotted as dashed curves, with the characteristic distance $\delta$ in the latter being computed from (8.7) for $u_{c}=1$ and from (8.15) for $u_{c}=0$.

dashed lines represent the asymptotic predictions to be obtained below for dense and dilute sprays. As expected, vaporization is enhanced for larger values of the coflow temperature, so that the value of $x_{v}$ decreases for increasing values of $T_{c}$ for both $u_{c}=0$ and $u_{c}=1$. 


\section{The limit $\lambda \gg 1$}

For sufficiently dense sprays with $\lambda \gg 1$, the vaporization front moves slowly, causing the resulting penetration distance to become much larger than the characteristic distance of jet development. The mixing layer between the emerging jet and the surrounding gas will be considered first, after which the subsequent development of the jet will be addressed.

\subsection{Mixing-layer solution}

Initially, the front is embedded in the mixing layer that departs from the injector rim, investigated above in $\S 6.3$. In this limiting case, the solution of (6.22) for $\eta<\eta_{v}$ is in the first approximation $F=\eta$, except in a thin layer $\eta \sim O\left(\lambda^{-1 / 2}\right)$ that need not be solved to obtain the solution for $\eta>\eta_{v}$, which is found by integrating (6.20) and (6.21) with boundary conditions $F_{\eta}-u_{c} / T_{c}=T-T_{c}=0$ at $\eta \rightarrow \infty$ and $F / 2=-T_{\eta} / \beta$ and $T-1=F_{\eta}-1=0$ at $\eta=0$. The resulting value of $F(0)=F_{+}$can be used in the first equation of (6.24) to obtain the vaporization-layer location according to

$$
\eta_{v}=\lambda^{-1} F_{+},
$$

where the constant $F_{+}$takes for $\beta=0.36$ and $T_{c}=(1.5,2.15,3.0)$ the values $F_{+}=(-0.6572,-1.0084,-1.2455)$ and $F_{+}=(-0.8228,-1.2062,-1.4408)$ for $u_{c}=0$ and $u_{c}=1$, respectively. On the other hand, the second derivative $F_{\eta \eta}(0)=\left(F_{\eta \eta}\right)_{+}$ can be used in (6.25) to obtain $\left(F_{\eta \eta}\right)_{-}=\left(F_{\eta \eta}\right)_{+}-\beta F_{+} / 2$, which in turn determines from (6.22) the small departures

$$
F-\eta=(\sqrt{\pi} / 2)\left(F_{\eta \eta}\right) \lambda^{-1} \mathbf{i}^{1} \operatorname{erfc}\left[-\lambda^{1 / 2} \eta /(2 \sqrt{P r})\right]
$$

of the streamfunction for $\eta<\eta_{v}$, where $i^{1}$ erfc is the first integral of the error function. The prediction for the initial front location given in (8.1) is found to be very accurate, as can be seen in the comparisons of figure 8 .

\subsection{Leading-order analysis}

The vaporization front continues to move slowly as the jet develops, with most of the spray vaporization occurring for $x \sim \delta \gg 1$, where $\delta(\lambda)$ is to be determined as part of the asymptotic analysis for $\lambda \gg 1$. The terms involving axial derivatives in (6.1)-(6.7) are of order $\delta^{-1}$, as is apparent when the rescaled coordinate $X=x / \delta$ is introduced. If the corresponding term is neglected in (6.5), integration with boundary condition $v=0$ at $r=0$ yields $v=0$ for $0<r<r_{v}$, which can be used in integrating (6.6) and (6.7), also with axial convection neglected, to give the uniform profiles $u=u_{v}$ and $Y=Y_{v}$ for $r<r_{v}$. Note that, across the spray, the departures of $v$ and $Y-Y_{v}$ from their leading-order values $v=0$ and $Y=Y_{v}$ can be expected to be of order $\delta^{-1}$, which is the relative error associated with the axial derivative that has been neglected in integrating (6.5) and (6.7). However, because of the small factor $1 /(1+\lambda)$ affecting the viscous force in (6.6), axial velocity variations $u-u_{v}$ in this region are much larger, of order $\lambda / \delta$, leading to a radial velocity gradient at the vaporization front given by

$$
\operatorname{Pr}\left(\frac{\partial u}{\partial r}\right)_{-}=\frac{\lambda}{\delta} \frac{1}{2} r_{v} u_{v} \dot{u}_{v},
$$

as can be seen by integrating once (6.6) for $u-u_{v} \ll 1$. Here, the dot will be used to denote differentiation with respect to the rescaled axial coordinate $X$, so that, for instance, $\dot{u}_{v}=\mathrm{d} u_{v} / \mathrm{d} X$ in the above equation.

The quasi-steady profiles obtained for $r>r_{v}$ by neglecting axial convection in (6.1)(6.4) provide the solution at distances $r-r_{v} \sim O(1)$ with small errors of order $\delta^{-1}$. 
Integration of (6.1) gives $\rho r v=-(\lambda / \delta) u_{v} r_{v} \dot{r}_{v}$, with the first equation in (6.13) with $v_{-}=0$ employed to evaluate the constant value of the radial mass flux. Using this result in integrating (6.3) with boundary conditions $T=1$ and $(\partial T / \partial r)_{+}=-\beta(\lambda / \delta) u_{v} \dot{r}_{v}$ at $r=r_{v}$ yields

$$
T=\beta\left(r / r_{v}\right)^{-(\lambda / \delta) u_{v} r_{v} \dot{r}_{v}}-\beta+1,
$$

whereas, at the same level of approximation, integration of (6.2) and (6.4) gives

$$
\frac{\lambda}{\delta} u_{v} \dot{r}_{v}\left(u-u_{v}\right)=-\operatorname{Pr}\left(\frac{\partial u}{\partial r}\right)_{+}\left[\left(1+\frac{T-1}{\beta}\right)^{1 / P r}-1\right]
$$

and

$$
Y=1-\left(1-Y_{v}\right)\left(1+\frac{T-1}{\beta}\right)^{L},
$$

when the boundary conditions $u=u_{v}$ and $\partial u / \partial r=(\partial u / \partial r)_{+}$and $Y=Y_{v}$ and $\partial Y / \partial r=(\lambda / \delta) u_{c} L\left(1-Y_{v}\right) \dot{r}_{v}$ are employed, the latter determined from (6.13) with $(\partial Y / \partial r)_{-}=0$.

The equations that determine $r_{v}(X), u_{v}(X)$ and $Y_{v}(X)$ are obtained by matching the quasi-steady profiles (8.4), (8.5) and (8.6) with those found in the far-field region, where the effect of axial convection can no longer be neglected in (6.1)-(6.4). For non-zero values of $u_{c}$, this region corresponds to radial distances of order $r \sim \sqrt{\delta}$, as follows from a simple convection-diffusion balance in (6.2)-(6.4). Therefore, matching at leading order requires that $T-T_{c}, u-u_{c}$ and $Y$ all be small at radial distances of order $r \sim \sqrt{\delta}$. When this condition is used in (8.4), the scaling law

$$
\lambda \ln (\delta) / \delta=1
$$

follows, along with the leading-order result

$$
u_{v} r_{v} \dot{r}_{v}=-2 \ln \Lambda
$$

where

$$
\Lambda=1+\frac{T_{c}-1}{\beta} .
$$

At the same level of approximation, (8.5) and (8.6) lead to

$$
2\left(u_{v}-u_{c}\right) \dot{r}_{v}=\left(\Lambda^{1 / P_{r}}-1\right) r_{v} \dot{u}_{v}
$$

and

$$
Y_{v}=1-\Lambda^{-L}
$$

with (8.3) used to evaluate $(\partial u / \partial r)_{+}=(\partial u / \partial r)_{-}$in deriving (8.10) from (8.5). Straightforward integration of (8.10) with $r_{v}=1$ when $u_{v}=1$ gives

$$
u_{v}=u_{c}+\left(1-u_{c}\right)\left(r_{v}^{2}\right)^{1 /\left(\Lambda^{1 / p r}-1\right)},
$$

which can be substituted into (8.8) to provide an evolution equation for $r_{v}(X)$, finally yielding

$$
4 X \ln \Lambda=u_{c}\left(1-r_{v}^{2}\right)+\left(1-\Lambda^{-1 / P_{r}}\right)\left(1-u_{c}\right)\left[1-\left(r_{v}^{2}\right)^{1 /\left(1-\Lambda^{-1 / P_{r}}\right)}\right]
$$

upon integration with initial condition $r_{v}=1$ at $X=0$. The rescaled penetration distance $X_{v}=\left[1+\left(u_{c}-1\right) \Lambda^{-1 / P_{r}}\right] /(4 \ln \Lambda)$ follows from setting $r_{v}=0$ in the above 
equation. At leading order, the asymptotic analysis therefore gives

$$
x_{v}=\frac{\delta\left\{1+\left(u_{c}-1\right) \Lambda^{-1 / P r}\right\}}{4 \ln \Lambda},
$$

as a prediction for the penetration distance when $u_{c} \neq 0$, with $\Lambda$ given in (8.9) in terms of $T_{c}$ and $\beta$ and $\delta \simeq \lambda \ln \lambda$ determined from (8.7) for a given value of $\lambda \gg 1$. This prediction is compared in figure 10 with the results of numerical computations of the sheath-vaporization problem for $u_{c}=1$, yielding excellent agreement over the range of $\lambda$ computed.

The length scale $\delta$ defined in (8.7) is modified when $u_{c}=0$, because convection in this case enters farther from the spray, in a region whose characteristic radius can be obtained from the convection-diffusion balance $r^{2} / \ln r \sim \delta$, obtained from (6.2)-(6.4) with $u \sim T-T_{c} \sim Y \sim 1 / \ln r$, a scaling that follows from the asymptotic decay of the quasi-steady profiles (8.4)-(8.6). As a result, at the order computed above, the equation that determines $\delta$ becomes

$$
\lambda \ln [\delta \ln (\delta)] / \delta=1,
$$

which should be used instead of (8.7) when $u_{c}=0$. It is easy to see that the rest of the development leading to (8.14) remains identical, so that the leading-order asymptotic prediction for the penetration distance of dense spray jets discharging into a stagnant hot atmosphere is given by (8.14), with $u_{c}=0$ and with $\delta$ computed from (8.15), equivalent to $\delta=\lambda \ln (\lambda \ln \lambda)$ at this order. This prediction is compared in figure $10(a)$ with results of numerical integrations. As can be seen, the resulting accuracy is reasonably good, with departures remaining smaller than $20 \%$ for the two values of $T_{c}$ considered, in agreement with the errors of order $(\ln \delta)^{-1}$ associated with the leading-order asymptotic development. It is worth pointing out that these differences in radial scale between the cases $u_{c} \sim O(1)$ and $u_{c}=0$ were also previously encountered in classical boundary-layer analyses in cylindrical geometries, with the scale for the case $u_{c} \sim O(1)$ corresponding to that found by Glauert \& Lighthill (1955) for the boundary layer developing over a stagnant cylinder and that of the case $u_{c}=0$ being related to that used by Crane (1972) in his analysis of a cylinder moving in a fluid at rest.

\subsection{Higher-order corrections}

The leading-order predictions for $Y_{v}, u_{v}$ and $r_{v}$ given in (8.11), (8.12) and (8.13) and the accompanying prediction for $x_{v}$ given in (8.14) can be improved by introducing expansions for the different variables in increasing powers of $(\ln \delta)^{-1}$. The analysis may employ the results $Y=Y_{v}$ and $v=0$ for $r<r_{v}$ along with the quasi-steady profiles given in (8.4), (8.5) and (8.6) for $0<r-r_{v} \sim O(1)$, because the associated errors are of order $\delta^{-1} \ll(\ln \delta)^{-1}$. Matching with the far-field solution beyond the order used in deriving (8.11)-(8.13) must be however considered, along with higher-order corrections to $(\partial u / \partial r)_{\text {- }}$ arising from convective effects for $r<r_{v}$, with the leadingorder result (8.3) being replaced by a more elaborate expression involving powers of $(\ln \delta)^{-1}$. As an example, results are given below for the case $u_{c}=1$, for which the required development is simpler, because the solution for the velocity field everywhere reduces to $u=1$, so that corrections to $r_{f}$ stem only from higher-order matching of the temperature field with the solution for $r \sim \delta^{1 / 2}$.

The analysis begins by writing the quasi-steady profile (8.4) for $r \sim \delta^{1 / 2}$ in the form

$$
\ln \Lambda+\ln \left(1+\frac{T-T_{c}}{\beta \Lambda}\right)=-\frac{1}{2} r_{v} \dot{r}_{v}\left[1+\frac{2}{\ln \delta} \ln \left(\frac{r}{\delta^{1 / 2} r_{v}}\right)\right] .
$$


The vaporization front $r_{v}$ is determined as an expansion of the form $r_{v}^{2}=A_{0}+$ $(\ln \delta)^{-1} A_{1}+(\ln \delta)^{-2} A_{2}+\cdots$ by matching the temperature profile given above with that encountered in the far field, yielding at leading order $\dot{A}_{0}=-4 \ln \Lambda$, which can be integrated with initial condition $A_{0}(0)=1$ to give

$$
A_{0}=1-4 X \ln \Lambda \text {, }
$$

corresponding to the leading-order result (8.13) with $u_{c}=1$.

Investigation of the solution in the far field is required to obtain the first-order correction $A_{1}$. Observation of (8.16) reveals that $T-T_{c} \sim(\ln \delta)^{-1}$ for $r \sim \delta^{1 / 2}$, which justifies the selection of the rescaled temperature

$$
\theta=\frac{(\ln \delta)\left(T-T_{c}\right)}{2 \beta \Lambda \ln \Lambda}
$$

for the analysis of the far field in terms of the rescaled radius $R=r /\left(T_{c} \delta\right)^{1 / 2}$, with the term $T_{c}^{1 / 2}$ included in the definition for convenience. The governing equation for the leading-order term in the expansion $\theta=\theta_{0}+(\ln \delta)^{-1} \theta_{1}+\cdots$ can be seen from (6.3) to be

$$
\frac{\partial \theta_{0}}{\partial X}-\frac{1}{R} \frac{\partial}{\partial R}\left(R \frac{\partial \theta_{0}}{\partial R}\right)=0,
$$

which must be integrated with initial conditions $\theta_{0}=0$ at $X=0$ and with boundary conditions $\theta_{0}=0$ as $R \rightarrow \infty$ and $R \partial \theta_{0} / \partial R=1$ as $R \rightarrow 0$, the latter following from matching with (8.16). The solution reduces to $\theta_{0}=-\frac{1}{2} E_{1}\left[R^{2} /(2 X)\right]$, where $E_{1}$ is the exponential integral (Abramowitz \& Stegun 1965), with the simplified form

$$
\theta_{0}=\ln R+\frac{1}{2}[\gamma-\ln (2 X)]
$$

applying as $R \rightarrow 0$, where $\gamma$ is Euler's constant. Matching (8.20) with (8.16) gives $\dot{A}_{1} / \dot{A}_{0}=\gamma-\ln \left(2 X T_{c} / A_{0}\right)$, which can be integrated with initial condition $A_{1}(0)=0$ to provide the first-order correction:

$$
A_{1}=\left(A_{0}-1\right)\left[\gamma-\ln \left(2 T_{c}\right)-\ln X\right]+A_{0} \ln A_{0} .
$$

Solving now $r_{f}^{2}=A_{0}+(\ln \delta)^{-1} A_{1}=0$ for the first two terms in the penetration-distance expansion $X_{v}=X_{v 0}+(\ln \delta)^{-1} X_{v 1}+\cdots$ yields $X_{v 0}=1 /(4 \ln \Lambda)$ and

$$
X_{v 1}=-X_{v 0}\left[\gamma-\ln \left(2 T_{c}\right)-\ln X_{v 0}\right] \text {, }
$$

with the former corresponding to the leading-order result (8.14) with $u_{c}=1$. The expansion for $X_{v}$ can be used to write

$$
x_{v}=\frac{\delta}{4 \ln \Lambda}\left\{1-\left[\gamma-\ln \left(T_{c} / 2\right)+\ln (\ln \Lambda)\right] / \ln (\delta)\right\},
$$

as a corrected prediction for $x_{v}$ when $u_{c}=1$. Results obtained with this expression are essentially the same as those shown in figure 10 for the leading-order predictions coming from (8.14) until $\lambda \simeq 30$ but agree slightly better with the exact solution at smaller values of $\lambda$. The accuracy of the corrected prediction is therefore comparable with that found at leading order, with differences between both expressions being small, because the factor $\gamma-\ln \left(T_{c} / 2\right)+\ln (\ln \Lambda)$ appearing in the logarithmic correction is not very large for the values of $T_{c}$ and $\beta$ investigated. Improved accuracy must rely on corrections of order $(\ln \delta)^{-2}$ and smaller, which could be computed by carrying on the present analysis to higher orders, a development not further pursued here and not strongly motivated, in that logarithms of large numbers are not often very large. 


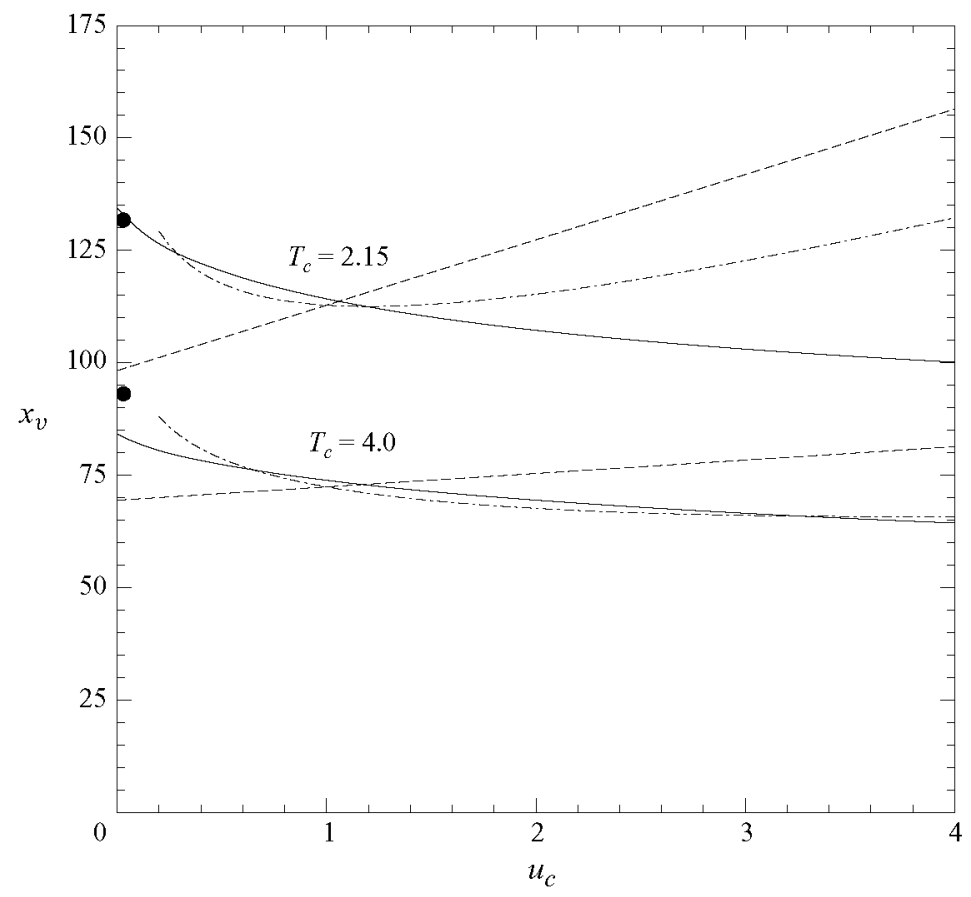

Figure 11. $(a, b)$ The spray penetration distance $x_{v}$ obtained in the limit $\varepsilon \rightarrow 0$ with $\beta=0.36$, $\operatorname{Pr}=0.7$ and $\lambda=100$ for $T_{c}=(2.15,4.00)$ and increasing values of $u_{c}$. The dashed and dot-dashed curves represent the leading-order prediction $x_{v}=[\delta /(4 \ln \Lambda)]\left[1+\left(u_{c}-1\right) \Lambda^{-1 / P_{r}}\right]$ and the corrected value $x_{v}=[\delta /(4 \ln \Lambda)]\left[1+\left(u_{c}-1\right) \Lambda^{-1 / P r}-\ln \left(u_{c}\right) / \ln (\delta)\right]$ with $\delta$ and $\Lambda$ evaluated from (8.7) and (8.9), while the solid dot denotes the prediction $x_{v}=[\delta /(4 \ln \Lambda)]\left[1-\Lambda^{-1 / \operatorname{Pr}}\right]$ for $u_{c}=0$, with $\delta$ evaluated from $(8.15)$.

\subsection{Influence of the coflow velocity}

As can be inferred from the comparisons in figure 10, the leading-order analysis describes satisfactorily the reduction in penetration distance associated with increasing values of $T_{c}$; as expected, increasing the coflow temperature produces a larger heat flux and therefore reduces the penetration distance, an effect clearly seen in the plots. The dependence of $x_{v}$ on $u_{c}$ is somewhat more complicated and deserves further attention.

The leading-order result for $u_{c} \neq 0$ given in (8.14) predicts a linear increase of $x_{v}$ with $u_{c}$. The increasing rate is however not very large for the small value of the latent heat of vaporization $\beta=0.36$ used here, because the accompanying factor $\Lambda^{-1 / P_{r}}$ is relatively small. This linear increase, due to spray acceleration, competes with a more subtle effect, coming from modifications to the radial heat flux, not accounted for in the leading-order prediction (8.14). As previously mentioned, in the limit of vanishing coflow velocities, the characteristic radius of the far-field region increases, which in turn reduces the radial heat flux reaching the spray, causing the characteristic spray length $\delta$ to increase from the value determined for $u_{c} \neq 0$ in (8.7) to the value given by (8.15). Since this additional effect is not accounted for in the leading-order analysis for $u_{c} \neq 0$, as $u_{c}$ is decreased the value of $x_{v}$ obtained from (8.7) and (8.14) approaches a limiting value below the asymptotic prediction for $u_{c}=0$, determined with use made of (8.15). This is seen in figure 11, which compares results 
of integrations of the sheath-vaporization problem for $\lambda=100$ and different values of $u_{c}$ with the asymptotic predictions for $u_{c}=0$ and $u_{c} \neq 0$.

As can be seen in the figure, the numerical integrations of the sheath-vaporization problem exhibit the increase of $x_{v}$ for decreasing $u_{c}$ discussed above, contrary to the prediction obtained by use of (8.7) in (8.14), which agrees with the numerical results only at values of $u_{c}$ appreciably larger than those of the figure. This effect can be captured in the asymptotic solution for $u_{c} \sim O(1)$ by incorporating corrections to $(8.14)$, of order $(\ln \delta)^{-1}$. Although the required analysis is not attempted here, it is relatively easy to extract the dependence on $u_{c}$ of the resulting correction term by studying the asymptotic development given in $\S 8.3$ for the special case $u_{c}=1$. As can be anticipated, in the modified analysis for $u_{c} \neq 1$, the far-field temperature solution should incorporate the value of $u_{c}$ in the definition of the radial coordinate $R=r /\left(T_{c} \delta / u_{c}\right)^{1 / 2}$. With this definition, the far-field equation for the temperature would reduce to (8.19) and matching the resulting solution with the inner quasi-steady temperature field would produce a term $-\ln \left(2 T_{c} / u_{c}\right)$ as a replacement for $-\ln \left(2 T_{c}\right)$ in (8.21) and also in (8.22). The associated correction $\ln \left(u_{c}\right) / \ln (\delta)$ can be incorporated when writing (8.14) to give $x_{v}=[\delta /(4 \ln \Lambda)]\left[1+\left(u_{c}-1\right) \Lambda^{-1 / P r}-\ln \left(u_{c}\right) / \ln (\delta)\right]$, with $\delta$ and $\Lambda$ evaluated from (8.7) and (8.9) respectively. The comparisons shown in figure 11 indicate that this corrected expression improves significantly the accuracy of the asymptotic limit $\lambda \gg 1$ over the range of $u_{c}$ shown in the figure, with the logarithmic correction providing the increase in $x_{v}$ found numerically as $u_{c} \rightarrow 0$. Additional analysis of the distinguished limit $u_{c} \sim 1 / \ln \delta$ could provide the transition between the asymptotic analyses for $u_{c} \sim O(1)$ and the limiting result for $u_{c}=0$ in dense sprays.

\section{The limit $\lambda \ll 1$}

For sufficiently dilute sprays with $\lambda \ll 1$, the amount of heat required to vaporize the spray and the resulting mass addition to the gas stream are both small, so that the solution for the gas temperature and fuel mass fraction is only weakly affected by the vaporization process. The heat flux coming from the coflow easily vaporizes the spray, yielding in the sheath-vaporization regime a vaporization front that propagates rapidly into the spray jet to complete vaporization at a short distance $x_{v} \ll 1$. Initially, the vaporization front lies on the innermost side of the annular mixing layer that forms downstream from the injector rim, at a location $-\eta_{v} \gg 1$. On the outer side corresponding to $\eta>\eta_{v}$, the solution is determined in the first approximation by integration of (6.20), (6.21) and (6.23) with boundary conditions $F_{\eta}-u_{c} / T_{c}=T-T_{c}=Y=0$ as $\eta \rightarrow \infty$ and $F-\eta=T-1=Y-Y_{j}=0$ as $\eta \rightarrow-\infty$, giving a temperature profile that decays towards the spray side according to

$$
T-1=-C \exp \left(-\eta^{2} / 4\right) / \eta
$$

where the constant $C$ is obtained as part of the integration, giving for $T_{c}=(1.5,2.15,3.0)$ the values $C=(0.1404,0.2764,0.4082)$ for $u_{c}=0$ and $C=(0.1948,0.3624,0.5275)$ for $u_{c}=1$, respectively. This vaporization-free solution fails as the vaporization front is approached for $\eta-\eta_{v} \sim-\eta_{v}^{-1} \ll 1$ where $T-1 \ll 1$ and $F-\eta_{v} \ll 1$. Introducing the stretched coordinate $\zeta=-\eta_{v}\left(\eta-\eta_{v}\right) / 2$ reduces the description of the temperature to the integration of $T_{\zeta \zeta}-T_{\zeta}=0$ with boundary conditions $T-1=T_{\zeta}-\beta \lambda=0$ at $\zeta=0$ and $T-1 \rightarrow-C\left[\exp \left(-\eta_{v}^{2} / 4\right) / \eta_{v}\right] \mathrm{e}^{\zeta}$ at $\zeta \rightarrow \infty$. Integrating once with the boundary conditions at $\zeta=0$ yields $T_{\zeta}-T=\beta \lambda-1$, and a second integration provides $T-1=-\beta \lambda-C\left[\exp \left(-\eta_{v}^{2} / 4\right) / \eta_{v}\right] \mathrm{e}^{\zeta}$. The condition $T=1$ 
at $\zeta=0$ then gives

$$
\beta \lambda=-C \exp \left(-\eta_{v}^{2} / 4\right) / \eta_{v},
$$

to determine $\eta_{v}$ as a function of $\beta$ and $\lambda$. The accuracy of this asymptotic prediction is very satisfactory, as can be seen in figure 8 .

As the vaporization front moves into the jet outside the annular mixing layer, effects of curvature enter to modify the heat flux that reaches the vaporization front from outside. In the intermediate region that lies between the mixing layer and the vaporization front, corresponding to radial distances such that $\sqrt{x} \ll 1-r<1-r_{v}$, the temperature and velocity differ by exponentially small amounts from the initial jet values $T=1$ and $u=1$, whereas the radial velocity is given simply by $v=\partial T / \partial r$, as can be seen by integrating (6.3) with $u=1$ and with $\lambda=0$ used in (6.13) when evaluating the boundary values $v_{+}$and $(\partial T / \partial r)_{+}$. Introducing this result into $(6.1)$ provides the linear heat equation

$$
\frac{\partial T}{\partial x}-\frac{1}{r} \frac{\partial}{\partial r}\left(r \frac{\partial T}{\partial r}\right)=0
$$

which is to be integrated with the boundary condition $T=1$ at $r=r_{v}$ and subject as $r \rightarrow 1$ to the matching condition with the mixing-layer solution given in (9.1). In the first approximation, the solution is given by

$$
T-1=C \sqrt{x}\left(\frac{\exp \left[-(r-1)^{2} /(4 x)\right]}{r^{1 / 2}(1-r)}-\frac{\exp \left[-\left(r_{v}-1\right)^{2} /(4 x)\right]}{r_{v}^{1 / 2}\left(1-r_{v}\right)}\right),
$$

with sample values of $C$ given below (9.1). Using now the additional boundary condition $\partial T / \partial r=-\beta \lambda\left(\mathrm{d} r_{v} / \mathrm{d} x\right)$ at $r=r_{v}$, obtained from (6.13) with $u_{v}=1$, provides

$$
\frac{C \exp \left[-\left(r_{v}-1\right)^{2} /(4 x)\right]}{2 r_{v}^{1 / 2} \sqrt{x}}=-\beta \lambda \frac{\mathrm{d} r_{v}}{\mathrm{~d} x},
$$

as an evolution equation for $r_{v}(x)$, which can be approximately solved for small values of $\lambda$ to give

$$
r_{v}=1-2\left\{x \ln \left[\frac{\lambda^{-1}}{\left[\ln \left(\lambda^{-1}\right)\right]^{1 / 2}}\right]\right\}^{1 / 2} .
$$

According to (9.6), the penetration distance for dilute sprays

$$
x_{v}=\left\{4 \ln \left[\frac{\lambda^{-1}}{\left[\ln \left(\lambda^{-1}\right)\right]^{1 / 2}}\right]\right\}^{-1},
$$

obtained from (9.6) with $r_{v}=0$, depends only on the parameter $\lambda$, all other parameters, including the velocity and temperature in the coflow, entering only in determining the higher-order corrections. Also of interest is that the modifications associated with curvature do not affect the solution at the order displayed in (9.7) in that the same prediction for the penetration distance is obtained by setting $r_{v}$ equal to zero in $\eta_{v}=\left(r_{v}-1\right) / \sqrt{x}$, derived above as the location of the vaporization front within the annular mixing layer that departs from the injector rim, with $\eta_{v}$ determined by solving (9.2) for $\lambda \ll 1$. The prediction (9.7) is tested in figure 9, giving good agreement for the different conditions considered. 


\section{U. Conciusions}

For a laminar, equilibrium, monodisperse fuel spray emerging steadily at a constant velocity from a round tube into a hot, chemically inert coflowing stream having a different constant velocity but the same molecular weight as the gas in the spray tube, the axisymmetric two-fluid conservation equations that account for finite-rate, diffusion-controlled evaporation and Stokes drag of spherical droplets in an ideal gas were integrated numerically to demonstrate explicitly the development of a regime of sheath vaporization as the ratio of the characteristic time of jet evolution associated with spray vaporization to the characteristic time for transverse diffusion across the jet approaches zero. This sheath-vaporization regime develops irrespective of whether the ratio of the mass of liquid to the mass of gas in the spray stream is large or small. If that ratio is large, then sample computations for octane sprays in air with a Lewis number of unity show explicitly that the fuel jet initially expands appreciably, its outer boundary being determined by the trajectory of the outermost droplet, until that droplet is completely vaporized, after which the outer boundary contracts, increasingly rapidly as the tip of the spray is approached. If, on the other hand, that mass ratio is small, then there is very little initial expansion of the jet, the shape of which now resembles a pointed icicle, much shorter than the jet for high liquid mass ratio because of the smaller amount of liquid to be vaporized.

In the limit of sheath vaporization, the initial expansion of the jet no longer occurs, there being a narrow vaporization layer, across which jump conditions are derived, connecting solutions of outer droplet-free differential equations to solutions of inner partial differential equations that describe the velocity and gas-phase fuelconcentration fields of the spray, the other variables there retaining their tube-exit values in the first approximation. The resulting free-boundary problem was also integrated numerically, making use of a mixing-layer solution near the injector rim, obtained numerically as well, to provide the necessary initial conditions for this parabolic problem. The numerical results give, for example, the jet penetration length as a function of the liquid-to-gas mass ratio of the spray for various ratios of coflow-to-spray temperatures and velocities, explicitly exhibiting the decrease in jet width and the increase in jet penetration length with increasing liquid-to-gas mass ratio.

Analytical formulae derived for the jet penetration distance in the dense-spray (large liquid-to-gas mass ratio) and dilute-spray (small liquid-to-gas mass ratio) limits agree reasonably well with the numerical results in those limits. In the dilute-spray limit, the penetration length is proportional to the product of the jet exit velocity and the transverse diffusion time, the proportionality constant depending only on the ratio of the liquid mass to the gas mass in the spray and increasing only weakly (inverse logarithmically) as this ratio increases. It is noteworthy that, in this limit, the penetration distance is entirely independent of the properties of the coflow stream at leading order, being controlled completely by the properties and dimensions of the lightly liquid-loaded spray.

If the coflow velocity is small enough, then the same proportionality of penetration length to the product of the jet exit velocity and transverse diffusion time occurs in the dense-spray limit as well (and therefore for all ratios of liquid-to-gas mass), but the proportionality constant increases much more strongly with increasing liquid-to-gas mass ratios (namely, in proportion to the product of this ratio with its logarithm) and, in addition, depends (relatively weakly) on the coflow temperature and the Prandtl number, decreasing as either of these increases. On the other hand, if the coflow velocity is sufficiently large, then in the dense-spray limit, the penetration 
distance is independent of the initial jet velocity but instead is proportional to the product of the coflow velocity and the transverse diffusion time, the proportionality constant again increasing more strongly with the liquid-to-gas mass ratio, and while it still decreases slowly with increasing coflow temperature, now its dependence on the Prandtl number is reversed. These last dependences, however, apply only for rather large coflow velocities, and at smaller coflow velocities the penetration length actually decreases with increasing coflow velocity, counterintuitively, as a consequence of a decrease in the coflow velocity producing an increase in the radial distance over which external heat conduction occurs, through reduction of entrainment, thereby decreasing the rate of heat transfer to the spray from the surrounding hot gas.

The results, in general, improve our knowledge of fuel-spray jet structure and penetration. Although formally restricted to steady laminar flow, qualitative interpretations for turbulent flows may be achieved by replacing the laminar viscosity by a turbulent viscosity, so long as the development of the spray is not significantly influenced by wall boundary layers and recirculation, for example. The regime of sheath vaporization, in particular, is often likely to be encountered in practice, and the present results may aid in insights into phenomena to be expected in that regime.

This work was supported by the Spanish MCINN through project CSD2010-00011.

\section{REFERENCES}

Abramowitz, M. \& Stegun I. A. 1965 Handbook of Mathematical Functions. Dover.

Aggarwal, S. K., Fix, G. J. \& Sirignano, W. A. 1985 Two-phase laminar axisymmetric jet flow: explicit, implicit and split-operator approximations. Numer. Meth. Partial Differ. Eq. 1, $742-756$.

ANNAMALAI, K. \& Ryan, W. 1992 Interactive processes in gasification and combustion. 1. liquid-drop arrays and clouds. Prog. Energy Combust. Sci. 18, 221-295.

Bermúdez, A., Ferrín, J. L. \& Liñ́n, A. 2007 The modelling of the generation of volatiles, $\mathrm{H}_{2}$ and $\mathrm{CO}$, and their simultaneous diffusion controlled oxidation, in pulverized coal furnaces. Combust. Theor. Model. 11, 949-976.

Blazek, J. 2001 Computational Fluid Dynamics: Principles and Applications. Elsevier.

Chen, G. \& Gomez, A. 1997 Dilute laminar spray diffusion flames near the transition from group combustion to individual droplet burning. Combust. Flame 110, 392-404.

Chiu, H. H., Ahluwalia, R. K., Koh, B. \& Croke, E. J. 1978 Spray group combustion. AIAA Paper 78-75 (presented at the 16th Aerospace Sciences Meeting, Huntsville, AL).

Chiu, H. H., Kim, H. Y. \& Croke, E. J. 1982 Internal group combustion of liquid droplets. Proc. Combust. Inst. 19, 971-980.

Chiu, H. H. \& LiU, T. M. 1977 Group combustion of liquid droplets. Combust. Sci. Technol. 17, $127-142$.

Correa, S. M. \& Sichel, M. $1982 a$ The boundary layer structure of a vaporizing fuel cloud. Combust. Sci. Technol. 28, 121-130.

Correa, S. M. \& Sichel, M. $1982 b$ The group combustion of a spherical cloud of monodisperse fuel droplets. Proc. Combust. Inst. 19, 981-991.

CRAne, L. J. 1972 Boundary layer flow on a circular cylinder moving in a fluid at rest. Z. Angew. Math. Phys. 23, 201-212.

Crowe, C., Sommerfeld, M. \& Tsuji, Y. 1998 Multiphase Flows with Droplets and Particles. CRC.

Dukowicz, J. K. 1980 A particle-fluid numerical model for liquid sprays. J. Comput. Phys. 35, 229-253.

Faeth, G. M. 1983 Evaporation and combustion of sprays. Prog. Energy Combust. Sci. 9, 1-76.

Glauert, M. B. \& Lighthill, M. J. 1955 The axisymmetric boundary layer on a long thin cylinder. Proc. R. Soc. Lond. A 230, 188-203.

Godsave, G. A. E. 1953 Studies of the combustion of drops in a fuel spray - the burning of single drops of fuel. Proc. Combust. Inst. 4, 818-830. 
Karpetis, A. N. \& Gomez, A. 2000 An experimental study of well-defined turbulent nonpreximed flames. Combust. Flame 121, 1-23.

KIм, H. Y. \& ChIU, H. H. 1983 Group combustion of liquid fuel sprays. AIAA Paper 83-0150 (presented at the 21st Aerospace Sciences Meeting, Reno, NE).

Labowsky, M. 1980 Calculation of the burning rates of interacting fuel droplets. Combust. Sci. Technol. 22, 217-226.

Labowsky, M. \& Rosner, D. E. 1978 Group combustion of droplets in fuel clouds. I. Quasisteady predictions. In Evaporation-Combustion of Fuels (ed. J. T. Zung), pp. 63-79. American Chemical Society.

Lasheras, J. C. \& Hopfinger, E. J. 2000 Liquid jet instability and atomization in a coaxial gas stream. Annu. Rev. Fluid Mech. 32, 275-308.

Lee, D., Kim, H. Y. Yoon, S. S. \& Cho, C. P. 2010 Group combustion of staggeringly arranged heptane droplets at various Reynolds numbers, oxygen mole-fractions, and separation distances. Fuel 89, 1447-1460.

LiÑ́́n, A. 1985 Theory of droplet vaporization and combustion. In Modélisation des Phénomènes de Combustion (ed. R. Borghi, P. Clavin, A. Liñán, P. Pelcé \& G. I. Sivashinsky), pp. 73-103 (CEA-EDF INRIA 59). Editions Eyrolles.

Luo, K., Pitsch, H., Pai, M. G. \& Desjardin, O. 2011 Direct numerical simulations and analysis of three-dimensional n-heptane spray flames in a model swirl combustor. Proc. Combust. Inst. 33, $2143-2152$.

ReVeilion, J. \& Vervisch, L. 2005 Analysis of weakly turbulent dilute-spray flames and spray combustion regimes. J. Fluid Mech. 537, 317-347.

Russo, S. \& Gomez, A. 2006 Physical characterization of laminar spray flames in the pressure range 0.1-0.9 MPa. Combust. Flame 145, 339-356.

Sichel, M. \& Palaniswamy, S. 1984 Sheath combustion of sprays. Proc. Combust. Inst. 20, 17891798.

SiRignano, W. A. 1983 Fuel droplet vaporization and spray combustion theory. Prog. Energy Combust. Sci. 9, 291-322.

SiRignano, W. A. 1999 Fluid Dynamics and Transport of Droplets and Sprays. Cambridge University Press.

Tanehill, J. C., Anderson, D. A. \& Pletcher, R. H. 1984 Computational Fluid Mechanics and Heat Transfer. Taylor and Francis.

Williams, F. A. 1985 Combustion Theory, 2nd edn. Benjamin Cummings. 